THESIS

\title{
EFFECTS OF FATIGUE ON BILATERAL GROUND REACTION FORCE ASYMMETRIES DURING THE SQUAT EXERCISE
}

\author{
Submitted by \\ Stephanie Hodges \\ Department of Health and Exercise Science
}

In partial fulfillment of the requirements

For the Degree of Master of Science

Colorado State University

Fort Collins, Colorado

Summer 2010 
COLORADO STATE UNIVERSITY

July 2,2010

WE HEREBY RECOMMEND THAT THE THESIS PREPARED UNDER OUR SUPERIVSION BY STEPHANIE JOLEEN HODGES ENTITLED EFFECTS OF FATIGUE ON BILATERAL GROUND REACTION FORCE ASYMMETRIES DURING THE SQUAT EXERCISE BE ACCEPTED AS FULFILLING IN PART REQUIREMENTS FOR THE DEGREE OF MASTER OF SCIENCE.

Committee on Graduate Work

Christian Puttlitz

Ray Browning

Advisor: Raoul F. Reiser II

Department Head: Gay Israel 


\section{ABSTRACT OF THESIS \\ EFFECTS OF FATIGUE ON BILATERAL GROUND REACTION FORCE ASYMMETRIES DURING THE SQUAT EXERCISE}

Physical performance and risk for injury have been related to functional asymmetries of the lower extremity. The squat exercise is an important, functional movement serving as the major multi-joint, closed-chain lower extremity exercise in many strength and conditioning programs, as well as a large component in rehabilitation. Examinations of asymmetries during the squat exercise have revealed bilateral differences in the net joint torques, indicating unequal actions between the lower extremities. To date, no study has examined how fatigue affects these asymmetries. PURPOSE: The goal of this investigation was to examine functional bilateral asymmetries of the lower extremities during the squat exercise, exploring how they may change during the course of an individual set, as well as across multiple sets with fatigue. Based on a review of literature related to the effects of fatigue on functional asymmetries, it was hypothesized that asymmetries would increase with fatigue. METHODS: Seventeen recreationally trained men $(n=9)$ and women $(n=8)$ (mean \pm SD age: $22.3 \pm 2.5$ yrs; height $170 \pm 9.3 \mathrm{~cm}$; mass $73.4 \pm 13.8 \mathrm{~kg}$ ) performed five sets of eight repetitions with $90 \%$ of their predetermined eight-repetition maximum (8RM $113 \pm 35 \%$ of their body mass). Anthropometric measurements and vertical ground reaction force (GRFv) data were collected. GRFv asymmetries were calculated by subtracting the \% load on the 
right foot from that of the left foot. Initially highly symmetric subjects $( \pm 1.7 \%)$ were removed from the analyses. The first two (R1\&2) and last two (R7\&8) repetitions of each set were examined. Eight subjects were reassessed for repeatability measures. RESULTS: R7\&8 took longer to complete than R1\&2 within a single set $(\mathrm{p}<0.001)$, however this finding was not observed across sets $(\mathrm{p}=0.867)$. The average asymmetry level across all sets for the absolute average was $4.3 \pm 2.5 \%$ and $3.6 \pm 2.3 \%$ for R1\&2 and $R 7 \& 8$, respectively. There was no difference in whole group analysis $(n=17)$ for any of the GRFv asymmetry analyses. Initially symmetric subjects were removed for the following analyses. For absolute peak instantaneous forces, the average asymmetry level across all sets was $3.8 \pm 2.3 \%$ and $3.2 \pm 2.3 \%$ for R1\&2 and R7\&8, respectively. Within a set, based on absolute asymmetry, levels of asymmetry dropped from beginning to end of a set $(\mathrm{p}=0.044)$. Exploring general shifts towards the left or right leg revealed a significant difference in peak instantaneous asymmetry with subjects becoming more symmetric with fatigue during the course of a single set $(\mathrm{p}=0.042)$. Of those who placed more weight on their right side at the beginning of a set, based on average asymmetry, a significant movement towards symmetry was seen for repetitions within a single set $(\mathrm{p}=0.036)$. Anthropometrics (segment lengths and circumferences) were generally not highly correlated to the asymmetries. Anthropometric measures were highly repeatable (Cronbach's $\alpha \geq 0.905, \mathrm{p} \leq 0.003$ ), as were time and asymmetry assessments of the squat GRFv asymmetries from visit to visit (Cronbach's $\alpha \geq 0.733, p \leq 0.056$ ). CONCLUSION: These results suggest that functional asymmetries though low were present, and remained consistent, in this healthy population during a squat exercise. Contrary to the hypothesis, asymmetries did not increase with fatigue, however time to complete each repetition 
within a set increased with fatigue. Healthy subjects appear to load limbs similarly, subjecting them to a relatively similar training stimulus.

Stephanie Joleen Hodges Department of Health and Exercise Science Colorado State University Fort Collins, CO 80523 Summer 2010 


\section{ACKNOWLEDGEMENTS}

First and foremost, I would like to thank my advisor, Raoul Reiser, for allowing this project to take place. His willingness to answer questions, edit documents, and offer guidance was more than I could hope for. I am very grateful for Dr. Reiser's interest in sponsoring an unfunded research project and treating it as if it were fully funded. I could not ask for a better thesis experience. This project could have also not taken place without my friend and fellow graduate student, Ryan Patrick, who was my co-pilot. Thanks to Ryan, there were many laughs to be had in the lab.

I would also like to thank the Health and Exercise Science department, including

all faculty and staff, for allowing me to complete my Master's degree and pursue a career change. My work at Colorado State University would not have been possible without a teaching assistantship, for which I am extremely appreciative. I cannot leave out my fellow graduate students, who offered their friendship, assistance, and comic relief.

Further, I would like to extend an enormous thanks to my family. Words cannot express how grateful I am of their never-ending support, encouragement, and love. I am truly blessed to have them in my life, especially my sister, who has been my personal cheerleader. I cannot leave out my husband, Jay, who propelled me to further my education and has been with me every step of the way offering guidance, encouragement, and acting as a general sounding board. Thank you, Love. 


\section{TABLE OF CONTENTS}

CHAPTER

$\underline{\text { PAGE }}$

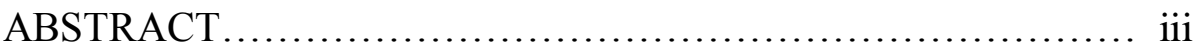

ACKNOWLEDGEMENTS ................................... vi

TABLE OF CONTENTS .................................... vii

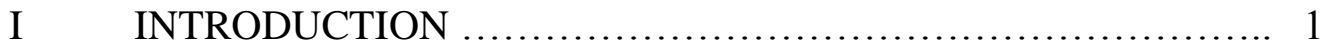

Statement of Problem $\ldots \ldots \ldots \ldots \ldots \ldots \ldots \ldots \ldots \ldots \ldots \ldots \ldots, 4$

Hypotheses....................................... 5

Limitations, Delimitations, and Assumptions............ 6

II LITERATURE REVIEW................................. 7

Development and Sources of Asymmetries................ 7

Geometric/Anthropometric..................... 8

Neural/Control................................ 10

Strength.................................... 12

Presence of Asymmetries............................... 14

Functional Evidence........................... 15

Performance................................. 17

Injury Risk.................................. 18

Fatigue........................................... 22

Functional Performance........................... 25

Fatigue and Asymmetries.............................. 27

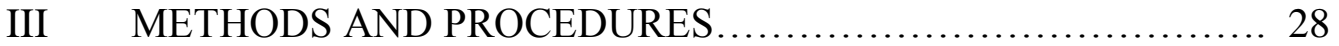

Experimental Approach to the Problem.................. 28

Subjects........................................ 29

Procedures............................................ 29

Data Processing and Analyses.......................... 33

Statistical Analyses.................................. 34 


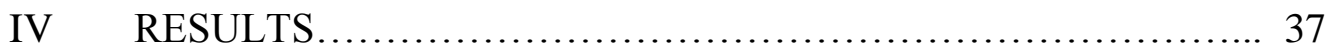

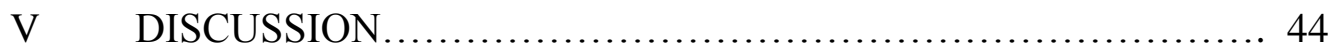

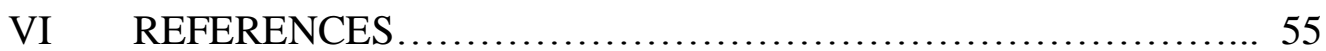

VII APPENDICES............................................. 61

Appendix A: Human Subjects Approval Form............. 62

Appendix B: Consent Form.......................... 64

Appendix C: Health and Activity Questionnaire........... 68 


\section{CHAPTER I}

\section{INTRODUCTION}

Physical performance and risk of injury have been related to functional asymmetries of the lower extremity. Functional asymmetries are related to the performance of a task asymmetrically, either kinematically, kinetically, or both. For instance, Tomkinson, Popovic, Martin (2003), suggested that those who are functionally symmetric also have improved physical performance. Asymmetric individuals then, may show decreased athletic performance or be at increased risk for injury due to favoring the stronger, or dominant side, of the body (Newton, et al., 2006). This finding is consistent with the discovery that asymmetry and poor alignment leads to increased energy expenditure and decreased muscular efficiency, which ultimately leads to increased stress and fatigue (Blustein, \& D’Amico, 1985). Asymmetries, such as strength imbalances in athletes, may be a result of training or various other factors, such as leg length discrepancies or previous injury (Newton et al., 2006). These asymmetries have been traced back to the womb in some cases (Blustein \& D’Amico, 1985).

While it would be expected to find asymmetries in injured populations, such as post-anterior cruciate ligament (ACL) reconstruction individuals (Ferber, Osternig, 
Woollacott, Wasielewski, \& Lee, 2004; Neitzel, Kernozek, \& Davies, 2002; Salem, Salinas, \& Harding, 2003), low levels of asymmetries have been recognized in otherwise healthy subjects. For example, functional asymmetries have been identified in a variety of tasks, such as jumping (Challis, 1998; Impellizzeri, Rampinini, Maffiuletti, \& Marcora, 2007; Lawson, Stephens, DeVoe, \& Reiser, 2006; Stephens, Lawson, DeVoe, \& Reiser, 2007), isometric knee extensions (Newton et al., 2006; Simon \& Ferris, 2008), static lifting and standing (Rocheford, DeVoe, \& Reiser, 2006), quiet stance (Blaszczyk, Prince, Raiche, \& Hebert, 2000), and the squat exercise (Flanagan \& Salem, 2007; Newton et al., 2006).

The squat exercise is an extremely important, functional movement serving as the major multi-joint, closed-chain lower extremity exercise in many strength and conditioning programs, as well as a large component in rehabilitation. This exercise incorporates major muscles of the lower back, hips, knees, and ankles; namely the gluteus maximus, hamstrings, quadriceps, and gastrocnemius (Escamilla, 2001; Gullett, Tillman, Gutierrez, \& Chow, 2008). Furthermore, since common activities such as the vertical jump, lifting of boxes, and sit-to-stand incorporate the joint actions of the squat, it may be a "bellwether" for many tasks.

Examinations of asymmetries during the squat exercise in healthy individuals have revealed bilateral differences in the net joint torques in recreationally trained men (Flanagan \& Salem, 2007), indicating unequal actions between the lower extremities. Net joint torques during a squat exercise were also examined under various loading conditions, as balance becomes an issue while free-weight squatting and may affect bilateral differences. For example, lighter loads may expose bilateral differences more, 
while heavier loads may cause one limb to generate greater torques in order to lift the heavier weight while maintaining balance (Flanagan \& Salem, 2007). However, the above-mentioned study failed to show that load affected bilateral differences. Additional bilateral differences have been observed in healthy collegiate women softball players performing a parallel back squat (Newton et al., 2006). They found no significant differences in peak force or average ground reaction force (GRF) between right and left legs, however significant differences were found between dominant and non-dominant legs.

Significant differences between dominant and non-dominant legs imply that a risk of differential development may unfold from uneven workout stimuli. Newton et al. (2006) hypothesized that specific strength training of the non-dominant leg may be beneficial as sports practice and competition may perpetuate strength imbalances. This discovery is especially important for those who participate in sports involving asymmetric movements for a prolonged period of time. For example, Lawson et al. (2006) demonstrated that the lead leg in a step-close jump incurs greater musculoskeletal demands than the trail leg in a group of recreationally competitive volleyball players. Fitness enthusiasts who lift weights on their own may also be of concern through improper lifting techniques, poor program design, and a host of individual factors.

One critical limitation of previous research is that assessment has occurred in the unfatigued state. It is unclear if this is a good representation of the state the activity is performed during the majority of a competition or workout. Knowledge of how asymmetries change with fatigue is extremely limited. Bilateral asymmetries increased with fatigue in a sustained isometric maximal voluntary elbow flexion contraction with 
right-handed oarsmen (Oda \& Moritani, 1995). It is however, uncertain if this static upper extremity protocol on an already asymmetric population translates to the squat. If it does, previously observed low levels of asymmetries might be more important than currently thought. Others have examined the effect of fatigue on asymmetry; discovering that asymmetries increased with fatigue in a swim bench exercise (Potts, Charlton, \& Smith, 2002). However, both of these groups had preferential development of one side of the body compared to the other based on their sport (oarsmen and swimmers that breathed to only one side). No studies examining fatigue relative to asymmetries have been conducted on groups without a predisposition. However, if level of effort is assessed (which could be associated with fatigue since maximum force declines with fatigue) it has been found that asymmetries remain constant (Flanagan \& Salem, 2007) or decline slightly (Carpes, Rossato, Faria, \& Bolli Mota, 2007). Furthermore, it has been found that speed of movement may be associated with asymmetry level with faster movements more asymmetric (Simon \& Ferris, 2008). Therefore, it is unclear how fatigue will affect functional GRFv during the free-weight squat exercise.

Additionally, if asymmetries increase with fatigue, the differential workout stimulus between legs may also be greater than originally anticipated, thus promoting the further development of asymmetries. Furthermore, fatigue has been associated with an increased risk for injury (Pappas, Sheikhzadeh, Hagins, \& Nordin, 2007). For instance, neuromuscular fatigue may bring about motor control and coordination adaptations, which have been thought to increase the risk for low back pain (Sparto, Parnianpour, Reinsel, \& Simon, 1997). Specifically, trunk movement patterns were altered, as evidenced as a result of fatigue, during a repetitive lifting task (Sparto et al., 1997). 
McLean \& Samorezov (2009) also demonstrated that central fatigue is a key player in ACL injury risk upon fatigue-induced landing. Unilateral fatigue led to a fatigue crossover to the contralateral limb when performing single-leg landings, which altered landing strategies (McLean \& Samorezov, 2009). If asymmetries increase with fatigue, they may then partially explain this increase in injury risk. Therefore, the goal of this investigation was to examine functional bilateral asymmetries of the lower extremities during the squat exercise in the context of how they may change during the course of an individual set, as well as across multiple sets. These results will then serve as a basis to limit the possible progression of asymmetric performance, as well as minimize the risk for injury.

\section{Statement of Problem}

The purpose of this study was to determine changes in lower extremity functional asymmetries with a fatiguing squat protocol as measured by GRFv in a group of healthy, recreationally trained males and females. Comparisons of anthropometric measures with GRFv were also made along with an examination of test re-test reliability.

\section{Hypotheses}

Based on the complete review of the literature that follows, it was hypothesized that individuals would be asymmetric (placing slightly more weight on one limb than the other) at the start of the squat protocol and that the degree of asymmetry would increase with fatigue. It is further hypothesized that the non-dominant stance leg would yield higher GRFs with fatigue and that anthropometric measures would have no relation to GRFs observed. It was also hypothesized that these methods would be reliable means to 
assess functional asymmetries (i.e., they will be consistent within sets, across sets, and from day-to-day).

\section{Limitations, Delimitations, Assumptions}

All subjects will be between the ages of 18 and 30 years. Subjects will have at least eight weeks of previous experience with the free-weight barbell squat, as incorporated into their own resistance training program, and will have been utilizing the jump in weekly sporting activities. Subjects that have lower extremity injuries or abnormalities resulting in known asymmetries will be excluded. Pregnant women will also be excluded. Limitations of this study include accuracy of anthropometric measurements due to difficulties in accurately measuring minute differences and measurements taken by two different researchers. During the screening process, we assumed the subjects reported correct information regarding their health, and physical activity. Also assumed is that subjects will perform at their maximal effort in all tests and procedures, not critically favor one side over the other, as well as adhere to study guidelines pertaining to abstaining from ergogenic aids and lower body exercise 48 hours prior to their visit. 


\section{CHAPTER II}

\section{LITERATURE REVIEW}

Bilateral asymmetry is a term used to describe differences between left and right sides of the body. Their presence may be random (fluctuating) in appearance or the result of acute or chronic events. Acute and chronic actions could be the result of an injury followed by incomplete recovery, while chronic may also be the result of repeated performance of asymmetric tasks and the ensuing adaptations made by the body. The asymmetry may be geometrical/anthropometric, neural/control, or strength related. The goal of this literature review is to present the known evidence of bilateral asymmetries in these three areas and expand as to how they may affect function and/or performance, as well as increase the risk for injury. The focus will be directed towards the lowerextremity and squat exercises, though other regions and movements will be included where appropriate. Finally, implications related to fatigue will be examined.

\section{Development and Sources of Asymmetries}

As a result of one's interaction with the environment, random fluctuations deviating from bilateral symmetry can develop (Trivers, Manning, Thornhill, Singh, \& McGuire, 1999) and appear in the form of imbalances from one side of the body to the 
other. These are known as fluctuating asymmetries with proposed sources in anthropometric, neural, or strength differences between the left and right sides of the body (Rocheford et al., 2006). Performing a task asymmetrically (either kinematically, kinetically, or both), resulting in a functional asymmetry (Rocheford et al., 2006), is likely, as a dominant limb that produces more force and does more work has been established in individuals (Newton et al., 2006). It is also reasonable for individuals to develop a foot preference, such that one limb is used to lead or manipulate objects and the other is used to provide postural support and thus the "mobilizing" limb is the dominant or preferred limb and the support limb becomes the non-preferred limb (Gabbard \& Hart, 2001). This functional separation may then encourage uneven use of the lower extremities and skill development (Stephens, Lawson, DeVoe, \& Reiser, 2007). It is necessary to state before proceeding further that an asymmetry is considered to be any deviation from zero (i.e., perfect symmetry) and is not clearly defined in the literature in terms of numerical value. For example, it would not be appropriate at this time to state a 2, 5, or $10 \%$ difference between sides defines someone as being asymmetric.

\section{Geometric/Anthropometric}

Leg length differences, an example of an anthropometric source, can themselves impact several variables, as Blustein \& D'Amico (1985) stated, there is decreased energy expenditure and increased muscular efficiency, leading to reduced stress and fatigue, with proper alignment and near symmetry. However, the majority of the population is not structurally symmetrical, such that one limb is longer than the other, averaging $1.1 \mathrm{~cm}$ upon maturity (Blustein \& D’Amico, 1985). Leg length inequality (LLI) is another term to describe this phenomenon, and has been associated with back pain, injury, and muscle 
strength asymmetry, as well as additional physiologic changes (Knutson, 2005). In Knutson's (2005) review, it was determined that $90 \%$ of the population is affected by some degree of LLI; however, this does not typically lead to problems if the difference is less than $20 \mathrm{~mm}$. For example, a leg-length difference of $28.6 \mathrm{~mm}$ had a substantial effect on oxygen consumption at a constant workload in a case study examining the effect of leg length difference on oxygen consumption (Delacerda \& McCrory, 1981). Leg length differences were suggested to be related to one's efficiency (Delacerda \& McCrory, 1981). Additionally, LLI and knee pathology were significantly correlated in an investigation by Kujala, Friberg, Aalto, Kvist, \& Osterman (1987) on lower limb malalignment and knee exertion injuries in male athletes with and without knee injuries. When jumping, the longer limb, more often, was determined to be the preferred takeoff leg, which helped to elucidate the relationship between LLI and knee exertion injuries (Kujala et al., 1987).

Furthermore, a 1970 paper regarding lower limb asymmetries contained observations made by Singh who concluded that most individuals do not use the lower extremities equally. Bone weight reflected the fact that one limb experiences enhanced development, as it is used more for bearing body weight when walking. White, Gilchrist, \& Wilk (2004) also reported differences in limb usage such that the shorter limb $(1-3 \mathrm{~cm})$ of individuals with a true or simulated LLI accepts more peak force and higher rates of loading than the longer limb. This was then found to predispose persons with LLI to musculoskeletal problems with repeated use (White et al., 2004). Bilateral asymmetries have also been examined using anthropometric measurements of the upper and lower limbs. For example, Krawczyk, Sklad, Majle, \& Jackiewicz (1998) measured 134 elite 
male athletes from nine different sports. These researchers suggested that asymmetries present in athletes are due to long-term, intense training and constant performance in sport-specific techniques and movements, which may result in bone or muscle hypertrophy differences between limbs. Athletes engaging in sports requiring symmetric movements displayed lower right-left differences in anthropometric measurements compared to athletes playing sports that utilize asymmetric movements in the abovementioned study. Additionally, cross-asymmetry, or the right arm and left leg being more developed for example, was also examined at the same time, however none was observed in the nine different sports studied.

Additional studies exploring asymmetries in individuals with the use of anthropometric measurements have failed to find any relation between the two (Lawson et al., 2006; Tomkinson, Popovic, \& Martin, 2003). However a recent examination on habitual throwing and swimming upon diaphyseal strength of upper limbs in cricketers and swimmers revealed that greater mechanical loads placed upon upper limbs, whether unilaterally or bilaterally, resulted in greater strength compared to less mechanical load (non-dominant limb or controls) (Shaw \& Stock, 2009). This finding demonstrates how repetitive activity or sport may lead to asymmetries within the body, however, whether this finding translates to the lower limbs is unknown.

\section{Neural/Control}

Neural asymmetries may arise due to neural or control drives and can be seen in handedness/footedness research. For instance, Gabbard \& Hart (1996) stated that one hemisphere dominants the other in respect to preferential use and skill, even though an 
individual can use both sides of the body fairly well. This has been evidenced in various studies revealing general dominance of the left-hemispheric control for motor asymmetries with contralateral control, in that most individuals are right-footed for motor skills and left-footed for stabilizing tasks (Gabbard \& Hart, 1996). Blaszczyk, Prince, Raiche, \& Hebert (2000) also concluded that motor tasks (locomotion initiation, kicking, and jumping) are dominated by one leg, and noted that the body is not symmetrical, neither in anatomical nor physiological conditions. Able-bodied gait has been found to be asymmetric, which may be the result of different contributions of the lower limbs in carrying out tasks (Sadeghi, Allard, Prince, \& Labelle, 2000). However, Singh (1970) concluded that that no distinct correlation between leg dominance and handedness could be made in his observation of lower extremity asymmetries. Not surprising then is the research conducted by Simon \& Ferris (2008) which revealed that limb asymmetry was related to neural factors, not differences in mechanical capabilities of the lower limbs.

Gait variables have been studied in basketball and soccer players, as well as swimmers to determine if playing a specific sport has an influence on gait pattern, thus leading to an asymmetric gait (Leroy, Polin, Tourny-Chollet, \& Weber, 2000). Differences in gait pattern between the left and right sides were observed, as were differences from sport to sport. However, differences were unable to be attributed to either asymmetric muscle development, a consequence of the central motor program, or a combination of both.

Further, asymmetries may be the result of bi-lateral deficit, which was reported by Challis (1998) in a study examining seven females maximally jumping from either their preferred jumping leg or both legs, with no countermovement. Bi-lateral deficit refers to 
a reduction in force production that is less than the sum of uni-lateral force productions, as a result of neural drive. Observed differences were a result of bi-lateral deficit in that differences in jump height existed between one- and two-legged jumps. However, coordination/control of this complex movement may have also been a contributing factor to the observed asymmetries.

Strength

Muscle strength imbalances may develop in part due to handedness, previous injury, or repeated use, as in training for a specific sport (Newton et al., 2006). Further, imbalances may either be strength differences between the left and right sides, or ratio differences between antagonistic muscle groups (Knapik, Bauman, Jones, Harris, \& Vaughan, 1991). It is also reasonable to conclude that strength differences between the left and right sides of the body may result from differences in contralateral groups of the same muscles or of different muscles within the limb.

Hewett, Stroupe, Nance, \& Noyes (1996) utilized a jump-training program to test the effects on landing mechanics and lower extremity strength in females that participate in jumping sports. Eleven female high school volleyball players, reported as right-hand dominant, were studied in which variables were compared to male athletes before and after their participation in the jump-training program. Force analysis tests were conducted on two types of jumps, performed five times each. In regards to leg dominance, hamstring muscle strength differences existed between the dominant and non-dominant leg. A difference between sides was also observed for hamstring-quadriceps peak torque 
ratio. Additional studies of drop jumps and jump landings have been explored in the context of fatigue and injury risk, and thus will be discussed below.

Soccer players have also been studied to examine asymmetries of the lowerextremities, as these athletes often show a dominance of kicking ability for one limb (McLean \& Tumilty, 1993). Twelve elite Australian junior soccer players were examined in a study by McLean \& Tumilty (1993) in which each subject performed two types of soccer kicks on each leg; a low drive kick and a chip kick. Knee extension and flexion strength for each leg was measured with a dynamometer at various settings. Kick velocity, kick accuracy, plant foot position from the center of the ball, and time from foot plant to contact with the ball was measured for each kick. Kick drive performance was better on the right leg, observed by drive velocity and accuracy, as was knee extension and flexion as a group, clearly indicating right leg strength dominance of these athletes. No difference was seen among measured parameters for chip kicks between the left and right legs, which the authors concluded was a difficult shot for both the left and right sides. In addition to observing strength differences between the left and right legs, some differences in kicking technique between each side was also observed. However, the authors suggest that further research is needed to understand how these variables affect kicking performance, but base their interest from a study (Starosta, 1988) examining shots at goal in high level competitions in which it was concluded that shooting symmetry is a necessary part of soccer training as elite athletes shoot for goal with both feet.

The squat has been used to identify asymmetries, in particular strength imbalances of the lower extremities (Newton et al., 2006) and differences in bilateral net joint torques 
(Flanagan \& Salem, 2007) in healthy populations in a non-fatigued state. Newton et al. (2006) examined 14 collegiate women softball players, who performed three sets of three squats at $80 \%$ of their one-repetition maximum with a two-minute rest between each set, and reported significant differences in peak force and average ground force between dominant and non-dominant legs, however no significant differences were found between right and left legs in the above mentioned variables. Flanagan \& Salem (2007) studied 18 recreationally trained men and women who performed three sets of three repetitions of the squat exercise under four load conditions with a three-minute rest between each set. Group analysis revealed significant bilateral differences in joint torques due to kinematics and ground reaction force kinetics that were significantly different from side-to-side, with a larger magnitude occurring on the left side. Further use of the squat exercise has been examined in patients recovering from anterior cruciate ligament (ACL) reconstruction to determine unequal loading responses between each limb (Neitzel et al., 2002; Salem et al., 2003). Both above mentioned studies reported significant bilateral differences between the involved (reconstructed) and uninvolved limb, lending important findings to rehabilitation issues. Additional discussion of the squat will occur later on, as will fatigue since the majority of studies examining asymmetries do so in an un-fatigued state, which does not address how fatigue affects asymmetries.

\section{Presence of Asymmetries}

Regardless of how asymmetries develop, the fact that they exist has been evidenced in a variety of tasks in mostly unfatigued states. Also apparent is the fact that asymmetries may then affect an individual's daily functioning and sport performance of athletes, as well as possibly lead to increased injury risk. For instance, Gupta, Nicholson, 
Shirley, \& Adams (2006) demonstrated that even though visual side-to-side symmetry was apparent during a $90^{\circ}$ squat, EMG muscle activation signals of the vastus lateralis were greater in the dominant leg. Therefore, this next section will focus on observed asymmetries in action and their affect on performance and injury.

\section{Functional Evidence}

Gait has also been studied to determine the effects of anterior cruciate ligament (ACL) deficiency and surgery on bilateral lower extremity joint adjustments compared to controls (Ferber, Osternig, Woollacott, Wasielewski, \& Lee, 2004). Interestingly, even the controls displayed hip joint asymmetry such that significant bilateral differences were observed in hip positive work and between-limb correlation coefficient values were low to moderate for hip moment and power curves. However, the authors argued that in healthy populations, gait asymmetry should not be considered pathological.

Jumping tasks have been the main method used to explore lower extremity asymmetries in action. Lawson et al. (2006) utilized 24 recreational volleyball players to explore biomechanical differences between the lead- and trail-leg during a step-close jump in order to contrast findings of each leg function in a no-step jump. Interestingly, anthropometric variables did not result in differences between the lead- and trail-legs, however kinematic and kinetic differences were observed between the two legs in a stepclose jump, which affected each joint to different extents. In the no-step jump, group differences between the two legs were observed (average ankle angle) that were not present in the step-close jump, indicating that additional individual mechanisms may be at work in generating asymmetries, such as from other tasks, pre-established leg 
dominance, or past experiences being injury, growth and development, and performance of daily living tasks (Lawson et al., 2006). Additionally, Stephens et al. (2007) examined 25 male and female college-aged volleyball players performing a series of jumping tasks and revealed that subjects jumped higher off one leg versus the other, but that degree of asymmetry greatly varied. The location of the biomechanical differences could not be determined, as significant findings in kinematic and kinetic data were not found.

Even the sport of cycling has been examined to explore effects of workload, pedaling rate and leg dominance upon pedaling asymmetry. Daly \& Cavanagh (1976) determined leg dominance and index of asymmetry (work done by the dominant extremity divided by work done by the other extremity, multiplied by 100) in 20 male recreational cyclists who then performed nine speed and resistance combinations, each one for three, thirty second trials, consisting of four testing situations which were completed in one day, and again on another day. Asymmetry in work output was reported, unrelated to limb dominance however. Changes in pedaling speed resulted in significant differences in asymmetry, yet a consistent trend did not exist for this relationship. There were many limitations in this study, however the authors conclude that asymmetry in racing cyclists may cause problems in that prolonged asymmetries may lead to early fatigue. Leg dominance, however, was reported to be responsible for the larger peak crank torque produced by the dominant leg in a group of six male sub-elite competitive cyclists (Carpes, Rossato, Faria, \& Bolli Mota, 2007). Further, this study determined that as crank torque and exercise intensity increased, pedaling asymmetries decreased. Conversely, Smak, Neptune, \& Hull (1999), established that pedaling 
asymmetry varied greatly among 11 subjects who pedaled at five different pedaling rates in order to understand asymmetry in cycle ergometry.

\section{Performance}

Physical performance has been positively associated with symmetry such that those who are symmetric exhibit superior physical performance (Tomkinson et al., 2003). This finding was based on a review of human and animal studies, however Tomkinson et al. (2003) failed to find a relationship between symmetry and adult male athletes from two different sports competing at two different levels. Specifically, symmetry was measured via anthropometric measurements in 52 elite and sub-elite basketball and soccer players from Australia and contrary to the hypothesis, researchers failed to see any differences in asymmetry between subjects from either sport or competition level. A control group was not examined, so whether or not differences exist between athletes and the general population is unknown from this study. As mentioned earlier, a case study exploring leg-length difference on oxygen consumption reported a substantial effect at a constant workload with varying leg-length differences (Delacerda and McCrory, 1981).

Looking purely at anthropometric measures to determine performance, Manning \& Pickup (1998) examined symmetry and athletic performance of middle distance runners. Fifty males participated wherein measurements were obtained for ear length, nostril width, finger digit length, and wrist width of both the right and left sides. Subjects also reported their best 800 - and 1500 -meter times. It was determined that the symmetric individuals ran faster than the asymmetric individuals with the best predictors of speed being nostril width and ear length. The authors concluded that this effect is not the result 
of a mechanical advantage to symmetry since measurements of the upper extremities were used. Conversely, a computer simulation study examining jumping performance with a either a zero or $10 \%$ bilateral strength asymmetry revealed minimal differences in jump height, as the strong leg compensated for the weak (Yoshioka, Nagano, Hay, \& Fukashiro, 2010). However, these authors stated that a bilateral strength asymmetry greater than $20 \%$ may lead to differences.

Valuable results of fatigue and performance not directly assessing the lower extremities can also provide insight. For example, external power output differed between the arms of ten competitive swimmers who completed four incremental swim ramp tests on a swim bench ergometer, which was greater for the left arm (Potts et al., 2002). Differences were also seen when the swimmers were separated into unilateral and bilateral breathers, with the bilateral breathers displaying an equally balanced power output between the arms. It is reasonable then to think that if differences exist for the upper extremities in regards to performance, they may then exist for the lower extremities.

\section{Injury Risk}

Determining asymmetries are important measurements that can be made to understand an athlete's increased risk for injury of the lower extremities either during training or competition (Impellizzeri, Rampinini, Maffiuletti, \& Marcora, 2007), as an increased risk for injury has been related to bilateral asymmetries (Arendt \& Griffin, 2000). Injury was addressed in the Tomkinson et al. (2003) study, in which it was determined that injury may lead to asymmetries appearing non-sided, which are not the 
result of developmental noise or stability. These concepts refer to a disruptive process (developmental noise) and a resistive process (developmental stability) an organism goes through as it seeks developmental precision, or bilateral symmetry (Tomkinson et al., 2003).

Measurements can also be made to assess whether an injured athlete is ready to return to their sport. The use of a vertical jump force test (VJFT) was established and determined to be a valid and reliable tool to measure bilateral strength asymmetry in healthy athletes and those recovering from unilateral knee surgery, which ultimately can be used in sports medicine (Impellizzeri et al., 2007). Specifically Impellizzeri et al. (2007) evaluated the VJFT which involved subjects performing a countermovement jump with both legs, hands on the hips, where one foot was on a force platform, the other on a leveled wooden platform. Jumps were alternated with the right or left foot on the force platform (five each leg) in which the vertical peak force produced by each leg on the platform was measured and the bilateral strength asymmetry was calculated. The VJFT data was then correlated with an isokinetic leg extension test and isometric leg press test performed by the same subjects in which significant correlations were found, thus establishing the validity of the VJFT. The usefulness of the VJFT, especially for those in rehabilitation programs, is that both legs perform the movement, thereby reducing the mechanical load on the injured limb, compared to the currently used one-leg hop test for functional assessment. Impellizzeri et al. (2007) even tested subjects 8-12 weeks after knee surgery and found the VJFT was well tolerated by the injured athletes and thus may be useful in the sport rehabilitation programs in order to monitor patients in the early stages of rehabilitation. Additionally, dynamic field tests have been examined in an NFL 
Combine setting in order to detect lower extremity asymmetries in athletes (Hickey et al., 2009). Specifically, the vertical hop power assessment and modified agility T-test both displayed reliable results that can aid in the determination of lower limb asymmetries and identify potential risk factors in these competitive populations (Hickey et al., 2009).

Knapik et al. (1991) assessed 138 female collegiate athletes from eight different sports through an extensive pre-season examination of leg strength and flexibility measures. It was determined that specific strength and flexibility imbalances were in fact associated with the first incidence of injury of the lower extremity in the female athletes. Higher injury rates were observed for those with imbalances of the knee flexor or hip extensor of $15 \%$ or more on either side of the body. Strains and sprains were more likely to occur on the left leg, which was determined to be the weaker side. As mentioned earlier, Hewett, et al. (1996) employed a six-week training program focusing on jumping and landing techniques, as well as increasing vertical jump height and strength. After training, the volleyball block jump revealed a decrease in peak landing forces of $22 \%$, while knee adduction and abduction moments decreased 50\%. A 26\% increase in hamstring-quadriceps muscle peak torque ratio was seen on the non-dominant side, while a $13 \%$ increase was seen on the dominant side upon training, which corrected side-to-side imbalances. Interestingly, training increased hamstring muscle power by $44 \%$ on the dominant side and only $21 \%$ on the non-dominant side. A statistically significant increase in mean vertical jump height after training was also seen. It was inferred that this jump-training program, which may prevent critical knee injuries in the female athlete, made a significant effect on knee stabilization. Discussion of improved strength imbalances between the sides was not discussed, but clearly this has implications for 
enhanced performance and reduced injury risk, as the reported methods would allow for pre-training and pre-competition detection of asymmetries.

Hewett, Lindenfeld, Riccobene, \& Noyes (1999) also suggest further studies to detect dominant versus non-dominant leg musculature, which may provide information about predisposition to injury for athletes. Such knowledge could also detect the need for pre-participation training. This recommendation came from their prospective study on the effects of neuromuscular training on the incidence of knee injury in female athletes, in which they observed a reduction in knee injury for females following a specific plyometric training program. Other sports activities have been examined to investigate imbalances, for example professional and junior level basketball players were studied to determine if lower limb explosive-strength asymmetries were present compared to each other and to a control group (Schiltz et al., 2009). An isokinetic knee extensor and flexor examination was performed, as was a countermovement jump, with and without arm movement, single-leg drop jump, a 10-m sprint, and 10-second single-leg continuous jumping. Both isokinetic and functional variables for professional and junior basketball players were similar, displaying no group dominant-side differences. The only bilateral asymmetries observed were for the professional level athletes on isokinetic and functional evaluations when previous knee injury was taken into account, such that the injured limb did not perform as well as the uninjured limb. These are interesting findings, as other researchers state that since basketball is somewhat a unilateral sport, most players have a preferential lay-up that is dependent on handedness (Leroy et al., 2000). 


\section{Fatigue}

The decline in a muscle's ability to produce force or perform work due to repeated or sustained contractions is known as fatigue, which also results in decrements in motor performance (Ewing \& Stull, 1984). Fatigue may be produced metabolically, or muscularly, which leads to a decline in performance. A change in coordination (neural input), a change in the muscles' functional ability to produce force, or a combination of the two may be the cause of the decline in performance (Rodacki, Fowler, \& Bennett, 2002). It has been shown that heavy resistance loading led to decreases in maximal neural activation and decreased explosive force production of the exercised muscles (Hakkinen, 1993), thus affecting performance. Various methods exist to quantify fatigue, which include alterations in joint kinematics, vertical ground reaction force magnitudes, or electromyographic activity (Smith, Sizer, \& James, 2009). Further, rate of perceived exertion, amount of force produced, or time to complete tasks may also be used to measure changes in fatigue. For example, an increased time to execute a task would indicate fatigue, as this would cause an inward shift on the force-velocity curve.

These are important concepts, as fatigue has been associated with athletic injuries (Pappas et al., 2007) whereby fatigue increases the risk of injury due to a loss of balance (Johnston, Howard, Cawley, \& Losse, 1998). Further, muscle fatigue leads to contractile force loss and muscle activation timing disturbance, which may then affect joint instability as was well as biomechanical alterations of the task (Vila-Cha, Carvalho, Machado, Conceicao, \& Vilas Boas, 2005). As such, researchers have sought to determine the effects of fatigue on various jump-related tasks, such as landing from a jump (Pappas et al., 2007; Smith, Sizer, \& James, 2009), performing a drop jump (Moran 
et al., 2009), performing a maximal vertical jump (Rodacki et al., 2002), and single-leg hop testing (Augustsson et al., 2006). Results have provided useful information on training programs to offset the effects of fatigue and improve landing technique, drop jump training, jump height performance, and functional performance when fatigued, all of which are especially important at the end of sporting events when fatigue is greater and injuries are more likely to occur (Dugan \& Frontera, 2000).

Specifically, Smith et al., (2009) studied 28 men and women to understand the effects of fatigue on multiple variables measured during drop-jump landings and determined that in fact fatigue affected frontal plane knee motion, increased EMG amplitudes, especially the hamstrings, and decreased ground reaction forces. Drop jumps from increasing heights were also used to examine impact acceleration, which was measured at the proximal tibia, under fatigued and non-fatigued conditions to assess injury risk (Moran et al., 2009). In addition, knee action during initial contact and landing phases were also studied. Moran et al. (2009) studied fifteen female soccer players who performed drop jumps from three heights $(15,30$, and $45 \mathrm{~cm})$ when fatigued and non-fatigued, which was induced by treadmill running. Impact accelerations and knee kinematics were recorded for each jump height in each condition. A significant interaction between fatigue and drop height on impact accelerations was observed, such that impact accelerations increased with jump height in the fatigued condition, however no significant difference was observed from the highest drop height of $45 \mathrm{~cm}$. A potential increased risk of injury when performing drop jumps under fatigue was demonstrated in that the neuromuscular system was negatively affected by endurance fatigue at drop jump heights of 15 and $30 \mathrm{~cm}$. Decreased neuromuscular control was evidenced by greater 
impact accelerations at greater heights and decreased knee angular velocities upon landing. The authors concluded that it may be safer or as safe to perform drop jumps from low(er) heights when fatigued than high(er) drop jump heights when non-fatigued and that coaches should be aware of the increased injury risk of performing such exercises under a fatigued state.

Subjects failed to jump as high when the knee extensors were fatigued compared to the non-fatigued condition as Rodacki, Fowler, \& Bennett (2002) sought to determine the decline in performance following fatigue using a vertical jump task performed with and without fatigue by 11 healthy, active subjects. Fatigue was induced by the use of a knee flexor/extensor weight machine at 50\% and $40 \%$ of body weight for the knee extensors and flexors, respectively. Three maximal countermovement jumps were performed in each condition where force data was used to determine the flight time of each jump.

Similarly, the squat has been used to study fatigue evidenced by Vila-Cha et al. (2005) who used the parallel half squat (PHS) to produce fatigue in 12 male subjects. EMG and kinematic data were recorded while subjects performed a PHS series for 92s (40 reps/min). Excitation of the motor neuron pool increased over time in order to maintain performance, demonstrating muscle fatigue was induced with prolonged PHS performance. Kinematic alterations were also observed on the movement pattern that was related to the increase in muscle activity. These two results were interpreted as biomechanical adjustments to muscle fatigue that may represent an increased injury risk factor. 
Load, cadence, and fatigue were measured in a study by Hattin et al. (1989) to determine effects on tibiofemoral joint force during 50 repetitions of the half squat that was performed by 10 males. Results of Hattin et al. study that are pertinent to the study at hand pertain to the effects of fatigue. Specifically, a significant effect on the magnitude of articular force components was shown due to fatigue, which was observed by differences between the initial and final, and the middle and final data collection intervals resulting in fatigue appearing during the middle phase and affecting the anteriorposterior shear force the most. The authors suggest that as a muscle fatigues, in order to produce the same amount of force, more muscle fibers are recruited, as are additional muscles in order to maintain stability of the knee, thus generating more overall muscle and joint force. This information is important to aid in the understanding of joint forces and their relationship with degenerative changes and injury, particularly for those who use the squat frequently in their training program.

\section{Functional Performance}

Tests for measuring dynamic sports function or rehabilitation outcomes generally assess subjects under non-fatigued conditions. Specifically, single-leg hops and vertical jumps are rarely performed for assessment in a fatigued state and thus fail to capture an athlete's physical-functional abilities when in a state of muscle fatigue (Augustsson et al., 2006), when injuries are more likely to occur. As such, literature surrounding the topic of muscle fatigue on functional performance is sparse. Therefore, Augustsson et al. (2006) sought to develop a single-leg hop test performed under fatigued conditions to assess reliability of such a measure and to examine kinetic and kinematic actions of the lowerextremity upon quadriceps muscle fatigue. Single-leg hop for distance with arm swing 
was performed by 11 healthy male subjects before and after a fatiguing exercise protocol which consisted of consecutive unilateral knee extensions using a load of $50 \%$ and $80 \%$ of one-repetition maximum (1RM) until failure was reached, whereby test-retest reliability was measured as the maximal hop length achieved under different conditions. Performance of hops under all test conditions resulted in satisfactory reliability of the test. Eight male subjects then performed the same protocol with the use of a threedimensional motion capture system and a force plate. The fatigued condition resulted in decreased hip and knee flexion angles, knee and ankle joint power, and ground-reaction forces during the single-leg hop take-offs when compared to the non-fatigued condition. The authors suggest that subjects took on a more up-right body position, decreasing the ability to generate horizontal forces when fatigued, as well as being less able to generate power than moment. Upon landing, hip moments and ground-reaction forces were reduced in the fatigued hop conditions, while the hip joint was more extended, especially after the fatiguing exercise using $80 \%$ of $1 \mathrm{RM}$. Thus, an adaptation was made by the subjects upon landing, via an extension of the hip. The fatigued condition also resulted in a significant decrease in single-leg hop distance due to quadriceps muscle fatigue, which explains the reduced ground reaction forces in that a shorter hop length reduces impact forces. Cleary fatigue affected the performance of the subjects, however this study resulted in a reliable test that can be used to investigate functional performance when fatigued and can be used to determine the effects of training or rehabilitation protocols. These researchers, however, did not directly compare left and right sides to see if fatigue affected them differently. 


\section{Fatigue and Asymmetries}

The above-mentioned studies exploring asymmetries have examined subjects in a non-fatigued state, which fails to determine how asymmetries change with fatigue. While fatigue has been studied, it has not been examined in the context of asymmetries to determine whether there is a change in level of asymmetry, or if one displays greater or lesser levels of asymmetries in a state of fatigue. As mentioned above researchers have shown that fatigue affects performance of various tasks and increases injury levels, yet how fatigue plays a role in asymmetry development is unclear. For example, if one limb fatigues faster than the other, it will not be developed the same from the exercise, as there will be a differential stimulus. For example, twenty-five right-handed oarsmen performed sustained maximal voluntary elbow flexion whereby the bilateral difference in fatigability was determined (Oda \& Moritani, 1995). The percentage of force decline in each arm was calculated and greater for the left arm compared to the right arm, thus the authors suggest that right to left differences exist in the fatigability of bilateral sustained maximal voluntary contractions. Similarly is the swim bench to exhaustion study discussed earlier that resulted in a greater magnitude of difference in bilateral arm power as subjects moved towards exhaustion (Potts et al., 2002). Granted these conclusions were based on findings of the upper extremities, it is possible that such differences hold true for the lower extremities as well. Further, not only does fatigue reduce maximal performance, if asymmetry increases with fatigue, that would compound the reduction. Additionally, not only does fatigue increase risk of injury, if asymmetry is increased, risk should increase as well. Based on this it is clear that further research is needed in this area. 


\section{CHAPTER III}

\section{METHODS AND PROCEDURES}

\section{Experimental Approach to the Problem}

To accomplish the goals of this investigation, a cross-sectional research design was used and approved by the Colorado State University Institutional Review Board (Appendix A). Subjects provided written informed consent (Appendix B) and visited the lab two-three times, in which data were collected all times. The first visit involved anthropometric measurements, familiarizations to the testing protocol, obtaining maximum single-leg hop distance on each leg, and determination of eight repetition maximum (8 RM) for the free-weight barbell back squat. During the second visit, subjects completed five maximal vertical jumps followed by three $50 \mathrm{~cm}$ single-leg horizontal hops on each leg. Five sets of eight repetitions $(5 \times 8)$ of the squat with a threeminute rest between each set were completed at $90 \%$ of the subject's predetermined 8 RM. The jump tasks performed prior to squatting were performed again immediately after completing the squat protocol. In both visits, subjects were allowed ample warm-up and stretching opportunities prior to familiarization with the protocol and data collection. The focus of this analysis was on the $5 \times 8$ squat protocol. 


\section{Subjects}

Subjects $(\mathrm{n}=17)$ were relatively fit $\left(\mathrm{BMI}<30 \mathrm{~kg} / \mathrm{m}^{2}\right)$, healthy individuals aged 18-30 years recruited mainly from the Colorado State University student population. Both men and women (approximately equal numbers of both) were studied and identified as recreational athletes that had been involved in resistance training programs whereby the squat had been incorporated for the past eight weeks (at minimum) and were currently involved in weekly sporting activities that utilized the jump (basketball, volleyball, racquetball, etc.). Exclusion criteria included self-reported pain, injury, and soreness at the time of each visit. Any injuries must have healed at least four weeks prior to participation and those with a history of back and/or lower limb pain, major previous surgery, bone, joint, or muscular disorder, history of neurological/orthopedic dysfunction, or pain that would limit the ability to perform the squat or jumps correctly were excluded. Any subjects with a known reason to perform the activities asymmetrically were also excluded (e.g., limb length discrepancy, ACL reconstruction, bilateral corrective devices, highly trained in an asymmetric skill, etc.). Finally, women who were pregnant at the time of investigation were excluded.

\section{Procedures}

Prior to participation, subjects were contacted either over the phone or in person in order to complete a brief health and activity questionnaire (Appendix C). Two separate visits to the laboratory were made for all subjects, each lasting approximately 1 hour per visit. Subjects were instructed to abstain from heavy exercise of the lower limbs and back for 48 hours before each visit. Caffeine consumption was limited to normal daily intake and no other ergogenic aids were allowed for consumption during the day 
prior to the visit. All testing on individual subjects was conducted at approximately the same time of day to avoid the effects of diurnal variations in performance. Details of each of the two visits are outlined below:

Visit 1- Final eligibility of subjects was determined during this visit along with other key preliminary functions. If not already obtained, the subjects provided written University approved informed consent (Appendix B). They were given orientation of the laboratory environment by laboratory staff followed by collection of anthropometric measures, including thigh circumference (identified at the midpoint obtained from the thigh length and measured with the foot placed on a chair with hip and knee at $90^{\circ}$ ), calf circumference (identified at the widest part of the calf and measured in a relaxed standing position with the heel on the ground, toes up, and weight shifted to the opposite leg), thigh length (inguinal crease to proximal patella, leg in same position as thigh circumference measure), lower leg length (knee crease to lateral malleolus when standing), foot length, and total supine leg length (SUPLL, anterior superior iliac spine to medial malleolus) (Evans, 1994) for each leg. Standing height and body weight were also recorded. A five-minute warm-up was required for all subjects before additional physical exercise. Options included an upright or recumbent stationary cycle ergometer. Stretching was also allowed and determined by each individual subject with the provision that a stretch done on one side of the body must be performed on the other side. The stretching allowed in the warm-up was to allow each subject to comfortably prepare for maximal exertion. Three to five sub-maximal vertical jumps with hands on the hips were practiced, followed by determination of a subject's single-leg maximum hop distance, which included approximately five to six hops per leg. Proper squat form for each 
subject was evaluated in order to determine if subjects could properly perform the freeweight barbell back squat exercise using guidelines described in the National Strength and Conditioning Association Position Paper (Chandler \& Stone, 1991). Visual verification that the squat was performed correctly was made by laboratory staff who also corrected any technique flaws. Subjects were instructed to wear proper athletic clothing and shoes similar to what they would normally wear during exercise, and were told to wear comparable clothes on the following visit. A lifting belt was made available for those in which it was a part of their normal squat routine. In addition to a standard $22 \mathrm{~kg}$ Olympic-sized barbell and weight plates, a "cage"-type squat rack was used with safety pins adjusted to the bottom range of barbell travel (Figure 1). Determination of each subjects' 8 RM was established and defined as the weight that could be lifted no more than 8 times with acceptable form. To determine the $8 \mathrm{RM}$, after a squat specific warmup of one to two light sets, subjects began with a weight assigned by the investigator based on the individual's current weight training program. Subjects completed up to 10 repetitions of that weight, with the barbell placed wherever they felt it to be comfortable on their shoulders, and were then asked to stop. After a three-minute rest, the weight was increased by $5 \mathrm{lb}(2.26 \mathrm{~kg})$. This procedure continued until an $8 \mathrm{RM}$ was determined. If the initial load was too heavy and 8 repetitions could not be completed, the load was reduced accordingly for the next attempt. A 1" $(2.54 \mathrm{~cm})$ diameter plastic depth bar was positioned behind the subject at the lowest point of their squat to ensure each repetition was performed the same (Figure 1). The depth bar was attached to vertical uprights with Velcro. A successful repetition required the subject to touch the depth bar before 
initiating the up phase of the squat. Two spotters were utilized during all lift attempts, one on each end of the bar. Verbal encouragement was provided for all exercises. Visit 2- Completed within two weeks after visit one (except for two subjects who were affected by temporary data capture equipment failure). Ground reaction forces were sampled at $1,000 \mathrm{~Hz}$ during all tasks of the Jump Protocol and Fatiguing Squat Protocol (described below) with two commercial force-measuring platforms (model 4060-10, Bertec Corp., Columbus, $\mathrm{OH}$ ) mounted side-by-side and flush to the surrounding floor (Figure 1).

Jump Protocol- After a warm-up similar to their first visit, subjects stood with feet parallel (one foot completely on each force platform with feet approximately shoulderwidth, no stagger) and were instructed to look straight ahead, with their head erect and hands on their hips. Performance of one approved trial consisting of five continuous maximum effort vertical jumps without rest then commenced. Following this, subjects hopped (one leg take off, same leg landing) from one force platform to another at a distance of 20 inches $(50 \mathrm{~cm})$. Three approved trials on each leg, alternating right and left legs, were performed. These jumps were then completed again, immediately following the fatiguing squat protocol.

Fatiguing Squat Protocol- The same equipment, set up, and squat specific warm-up was used as described in the first visit. Research assistants loaded the squat bar with $90 \%$ of the subject's previously determined 8 RM. Subjects then performed five sets of eight repetitions with a three-minute rest between each set. A brief pause between repetitions was inserted via verbal coaching to ensure each repetition was completed before the next was initiated. 
Repeatability Assessment- In order to verify the reliability of this experimental design, eight subjects returned to the lab a third time to establish day-to-day repeatability of the anthropometric measures, single-leg maximum jumps, and symmetry performance of the Jump and Fatiguing Squat Protocol. A minimum of 48 hours from the second visit was required prior to the third visit, with no more than 2 weeks separating the two (expect for one subject who was affected by temporary data capture equipment failure). The $1 \mathrm{RM}$ squat repeatability has been previously established (Hennessy \& Watson, 1994; Sewall \& Lander, 1991), as have higher repetition maximums of other exercises (Hoeger, Hopkins, Barette, \& Hale, 1990).

\section{Data Processing and Analyses}

Vertical ground reaction forces (GRFv) were low-pass Butterworth $\left(4^{\text {th }}\right.$-order, recursive) filtered at $10 \mathrm{~Hz}$ to remove noise. The average and peak instantaneous GRFv under each foot for the first two and last two repetitions of each squat set were analyzed (Figure 1). The start of a repetition was set at the time when the total (sum of right and left) GRFv dropped below body weight plus bar weight. The end of a repetition was the time when the total GRFv returned to body weight plus bar weight upon completion of hip and knee extension. Individual foot GRFv were normalized by the total GRFv and converted to a percentage. In this way, a perfectly symmetric repetition would yield a value of $50 \%$ under each foot for both the average and peak GRFv. GRFv asymmetry level was then calculated as the left GRFv \% minus the right GRFv\%, so a value of $2 \%$, for example, would indicate $51 \%$ on the left foot and $49 \%$ on the right foot and $-2 \%$ would indicate $49 \%$ on the left foot and $51 \%$ on the right. It should be noted that the peak instantaneous asymmetry calculation was based off of each foot's instantaneous 
maximum, not the maximum of the total GRFv. The results from the first two repetitions of each set were averaged for both the average and peak instantaneous GRFv asymmetries, as were the last two, so that a representative GRFv asymmetry was generated for the beginning (R1\&2) and end (R7\&8) of each set. Calculation of both the average and peak instantaneous GRFv is important as the average GRFv provides a representation of forces throughout the duration of each repetition while the peak instantaneous GRFv, although variable, yields the highest force observed.

\section{Statistical Analyses}

In addition to compiling means and standard deviations for anthropometric and biomechanical data, several higher level comparisons were performed. First, repeated measures t-tests were conducted on all parameters comparing the bilateral anthropometric measures. 2x5 (initial/final repetitions x sets) repeated measures MANOVAs were conducted on time to complete each repetition as well as the level of GRFv asymmetry. Since the goal of the investigation was to assess change in asymmetry level, and those who started very symmetrically in R1\&2 of set one would not be expected to change (which was verified throughout examination of the results), those with an asymmetry between $\pm 1.7 \%$ were excluded. $\pm 1.7 \%$ was chosen relatively arbitrarily. It was a level that excluded highly symmetric individuals, yet left a reasonably sized sample set to be evaluated ( $\mathrm{n}=12$ for both average and peak instantaneous asymmetries). Since some subjects began with greater GRFv on the left side and some on the right, the first assessment was on the absolute level of GRFv asymmetry. Within this analysis, if a subject switched sides from their initial value, it was assigned a negative number. Additional MANOVAs were preformed on the left minus right percentage of asymmetry 
without manipulation (\%L-R). One was performed on the whole group (excluding symmetric subjects), the subset of those that started with more GRFv on their left foot to start R1\&2, and the subset of those with more GRFv on their right foot to start R1\&2. These MANOVAs were performed on both the average and peak instantaneous asymmetries. Correlations (Pearson's r) were made to examine relationships between the anthropometric measures and both the average and peak instantaneous GRFv asymmetry. Finally, intraclass correlations (Chronbach's Alpha) were utilized to establish day-to-day repeatability with those that performed the procedures (anthropometric measurements and squat protocol) a second time. GRFv asymmetry repeatability was assessed within the first set (both R1\&2 and R7\&8, individually) as well as within the overall averages combining R1\&2 and R7\&8 of all sets. For these correlations the anthropometric measures were normalized to the subject's standing height converted to a percentage and the overall averages of the GRFv asymmetries were utilized. Statistical tests were conducted in PASW version 18.0 (SPSS, Inc., Chicago, IL) with significance set at $\mathrm{p}<0.05$. 


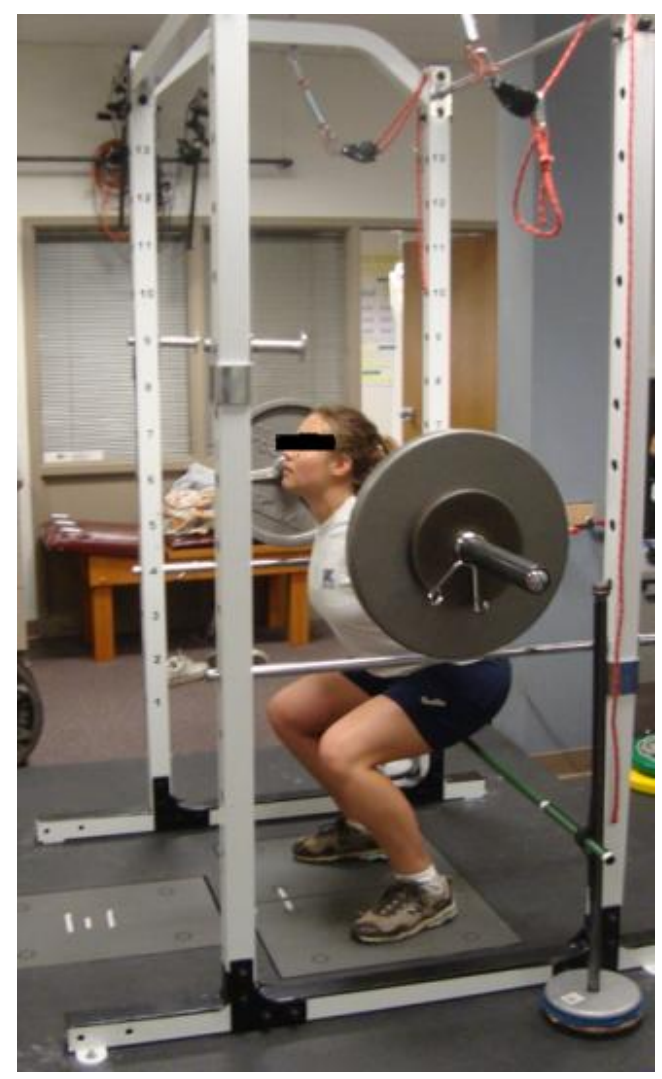

Figure 1: Squat cage positioned over force platforms with visible safety pins and depth bar. Also visible is the $3^{\text {rd }}$ force platform utilized for hoping tests. Note: Spotters normally stood at each end of the bar.

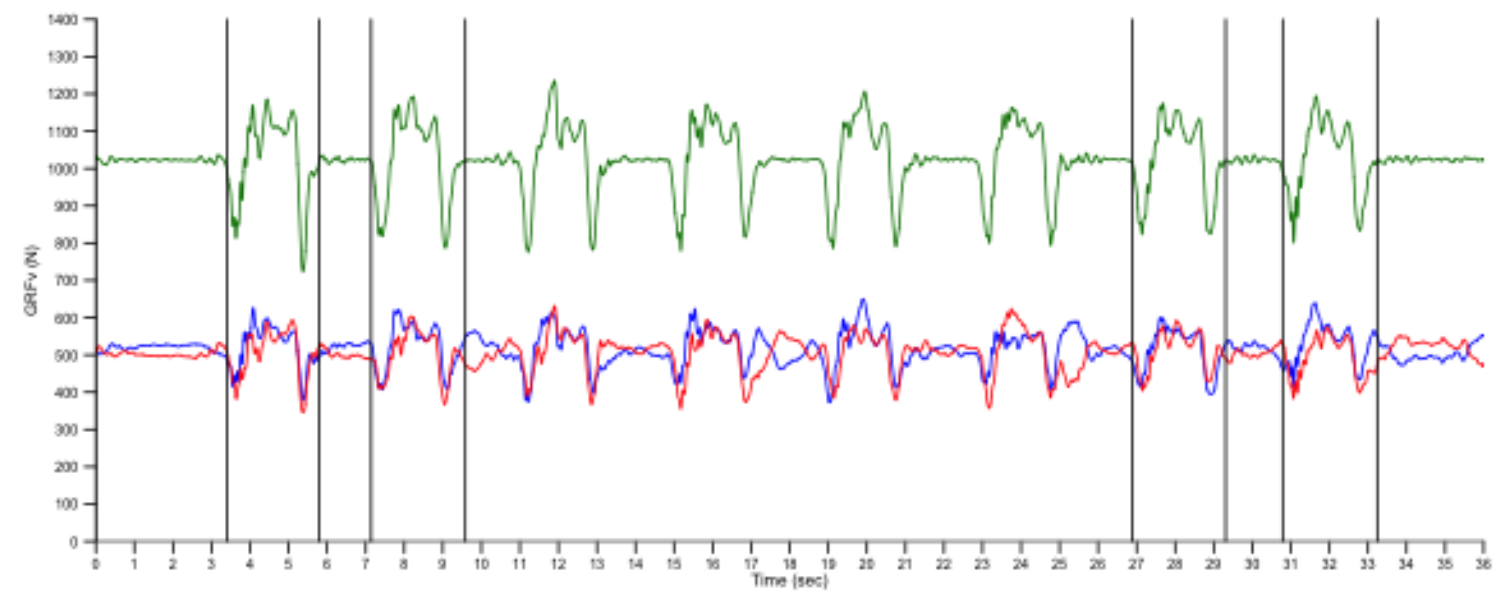

Figure 2: Exemplar Total GRFv (top line), Left GRFv (dark bottom line), and Right GRFv (light bottom line) for an 8 repetition set. Vertical lines identify the boundaries of repetitions 1,2, 7, and 8 . 


\section{CHAPTER IV}

\section{RESULTS}

Eight women and nine men completed the study (Table 1). Due to temporary data capture equipment failure, which postponed visits until equipment was repaired, two subjects between visit one to visit two and one subject from visit two to the repeat visit were forced to complete visits outside of the originally prescribed limits. This exaggerated the means for these variables slightly and greatly increased their standard deviations. Of the 17 subjects, eight (four men and four women) returned to complete the third visit to assess repeatability of the measurements. On average, subjects' 8RM was $113 \pm 35 \%$ of their body mass. All subjects reported their right leg and arm as their preferred kicking leg and throwing arm, respectively. There were no differences in anthropometrics between right and left sides of the body $(\mathrm{p}<0.001)$ (Table 2). All anthropometrics yielded significant correlations when comparing left to ride sides $(\mathrm{r} \geq 0.905, \mathrm{p} \leq 0.003)$

All subjects completed all repetitions of all sets at the $90 \%$ of their 8RM load. The 2x5 MANOVA assessing differences in time to complete each repetition $(\mathrm{R} 1 \& 2$, R7\&8), as well as each set (1-5), had no significant repetition by set interaction $(p=0.229)$ 
(Figure 2). However, there was a main effect for repetition with later repetitions taking longer to complete than initial repetitions within a single set $(\mathrm{p}<0.001)$. There was no main effect for set $(\mathrm{p}=0.867)$ in time to complete repetitions.

The absolute average of the whole group $(n=17)$ for R1\&2 of set one was $3.6 \pm$ $2.7 \%$ (range: 0.99 to $8.51 \%$ ) and $3.7 \pm 2.6 \%$ (range: 0.26 to $8.36 \%$ ) for R7\&8 of set one. In assessing the average \% L-R asymmetry, five subjects began R1\&2 of set one with more weight on their right leg, while 12 began on their left leg. Of these, eight subjects switched sides during at least one repetition/set data point. Within these eight subjects, three began R1\&2 of set one with an asymmetry level less than $1.7 \%$. The absolute peak instantaneous of the whole group $(n=17)$ for $R 1 \& 2$ of set one was $3.0 \pm 2.2 \%$ (range: 0.20 to $6.43 \%$ ) and $2.8 \pm 2.7 \%$ (range: 0.17 to $7.7 \%$ ) for $\mathrm{R} 7 \& 8$ of set one. In assessing the peak instantaneous \% L-R asymmetry, four subjects began R1\&2 of set one with more weight on their right leg, while the other 13 began on their left leg. Of these, nine subjects switched sides during at least one repetition/set point. Within these nine subjects, five began R1\&2 of set one with a peak asymmetry level less than $1.7 \%$.

Whole group analysis $(n=17)$ of average GRFv asymmetry failed to produce a significant repetition by set interaction $(\mathrm{p}=0.146)$. Significant main effects for repetition $(\mathrm{p}=0.600)$ or set $(\mathrm{p}=0.146)$ did not exist. Whole group analysis $(\mathrm{n}=17)$ of peak instantaneous GRFv also failed to produce a significant repetition by set interaction $(\mathrm{p}=0.622)$. Significant main effects for repetition $(\mathrm{p}=0.229)$ or set $(\mathrm{p}=0.414)$ did not exist. Thus, initially symmetric subjects $( \pm 1.7 \%)$ were removed from the following analyses as described in the Methods. 
As with time to complete repetitions, there were no repetition $\mathrm{x}$ set interactions in any of the analyses of GRFv asymmetries $(\mathrm{p} \geq 0.548)$. When categorized based on absolute asymmetry, where a reduction in value would indicate movement towards being more symmetric or possibly switching to the opposite leg they started with more weight on, there was a main effect for repetition $(\mathrm{p}=0.044)$ with absolute levels of asymmetry dropping from beginning to end of a set (i.e., they became more symmetric with fatigue), but not for set $(\mathrm{p}=0.530)$ (Figure 3a). When categorized based on peak instantaneous asymmetry, where again a reduction in value would indicate movement towards being more symmetric or possibly switching to the opposite leg they started with, there was neither a main effect for repetition $(\mathrm{p}=0.266)$ nor was there a main effect for set $(\mathrm{p}=0.349)$ (Figure 3b).

When examined to explore for a general shift towards the left or right foot in the whole group (less those that were initially highly symmetric), there were no significant main effects for repetition $(\mathrm{p}=0.788)$ or set $(\mathrm{p}=0.628)$ in the average $\% \mathrm{~L}-\mathrm{R}$ asymmetry (Figure 4a). However, there was a significant main effect for repetition $(p=0.042)$ in the peak instantaneous \%L-R asymmetry, where the group became more symmetric with fatigue, yet again there was no main effect for set $(p=0.266)$ (Figure $4 b)$.

When assessing those individuals who began with more weight placed on their left side $(n=9)$, based on average \%L-R asymmetry, significant main effects for repetition $(\mathrm{p}=0.212)$ and set $(\mathrm{p}=0.686)$ were not present (Figure 5a). The same analyses based on peak instantaneous \%L-R asymmetry of those beginning on their left side $(n=10)$ also failed to produce a significant main effect for repetition $(\mathrm{p}=0.080)$, though there was a trend towards becoming more symmetric with fatigue, but not set $(\mathrm{p}=0.199)$ (Figure $5 \mathrm{~b})$. 
When assessing those individuals who began with more weight placed on their right side $(\mathrm{n}=3)$, based on average \%L-R asymmetry, a significant main effect existed for repetition $(\mathrm{p}=0.036)$ with movement towards symmetry, but not for set $(\mathrm{p}=0.308)$ (Figure 6a). The same analyses based on peak instantaneous \%L-R asymmetry of those beginning on their right side $(\mathrm{n}=2)$ failed to produce significant main effects for repetition $(\mathrm{p}=0.540)$ and set $(\mathrm{p}=0.563)$ (Figure 6b).

In the correlations of overall average \%L-R asymmetry and overall peak instantaneous \%L-R asymmetry with left minus right anthropometric differences normalized to the subject's standing height, only the thigh length and calf circumference yielded significant correlations to overall average \%L-R asymmetry (Table 2). The only significant correlation for peak instantaneous \%L-R asymmetry was calf circumference. Intraclass correlations were completed on anthropometric measurements as well as data obtained from the squat protocol of the eight subjects who repeated the study. All anthropometric measurements taken from the left and right sides of the body, as well as height and weight were highly repeatable (Cronbach's $\alpha \geq 0.905, \mathrm{p} \leq 0.003$ ) (Table 3 ). Correlations of left minus right side anthropometric differences taken on different days only revealed a significant correlation for calf circumference $(\alpha=0.771, \mathrm{p}<0.035)$. All time and asymmetry assessments of the squat GRFv were highly correlated from visit to visit $(\alpha \geq 0.723, \mathrm{p} \leq 0.056)$ (Table 4).

Table 1. General Subject Characteristics

\begin{tabular}{lrr}
\hline & Mean & \multicolumn{1}{c}{ SD } \\
\hline Age $(\mathrm{yr})$ & 22.3 & $(2.5)$ \\
Height $(\mathrm{cm})$ & 170.8 & $(9.3)$ \\
Mass $(\mathrm{kg})$ & 73.4 & $(13.8)$ \\
Days between V1 \& V2 & 8.9 & $(9.1)$ \\
Days between V2 \& V3 & \multicolumn{1}{c}{12.6} & $(18.8)$ \\
\hline V1 = visit 1, V2 = visit 2, V3 = repeatability visit
\end{tabular}




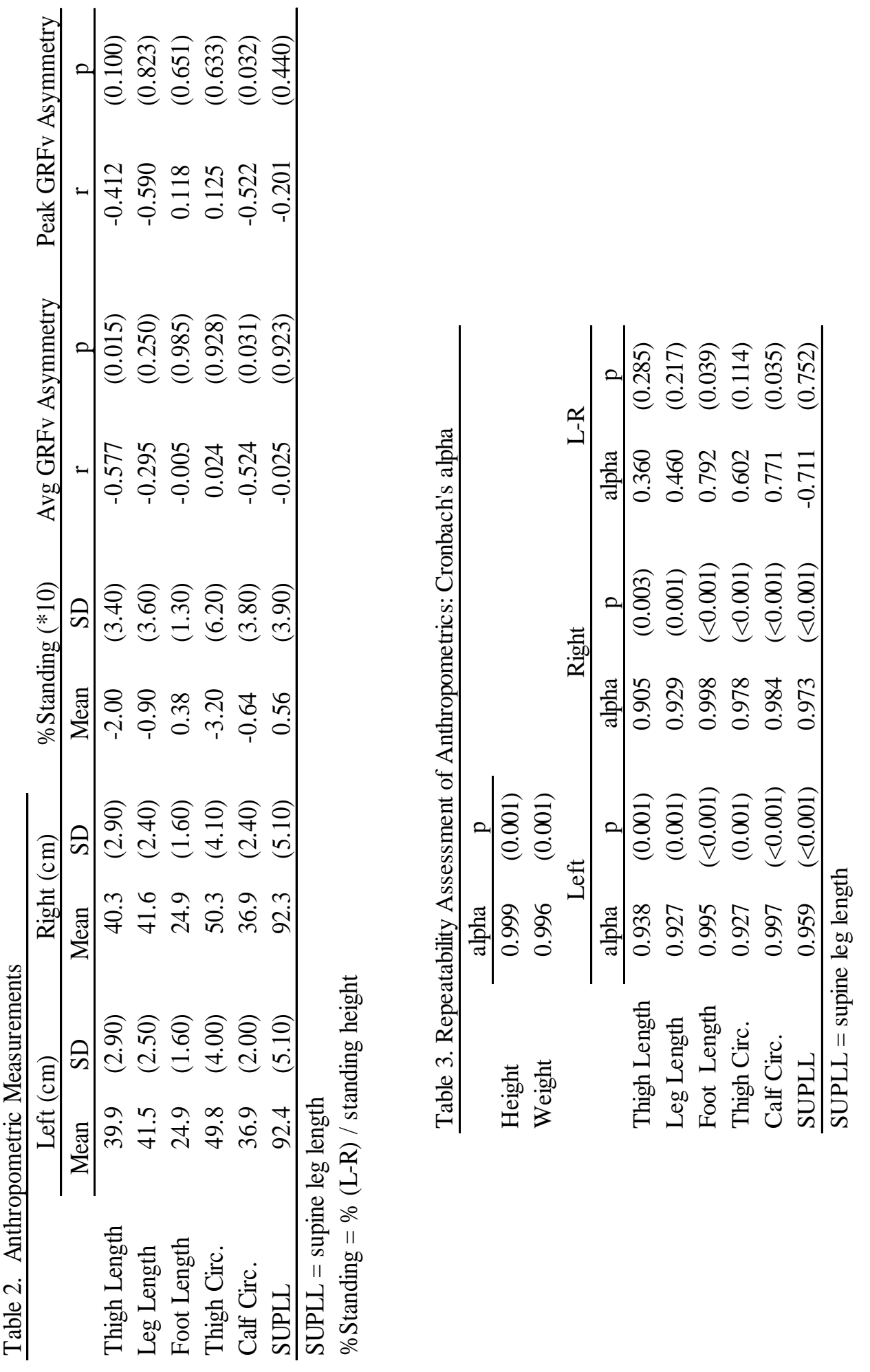


Table 4. Repeatability Assessment of Squat Protocol: Cronbach's alpha

\begin{tabular}{|c|c|c|c|c|}
\hline \multirow{5}{*}{$\begin{array}{l}\text { Overall Time R1\&2 } \\
\text { Overall Time R7\&8 }\end{array}$} & alpha & $\mathrm{p}$ & \multirow{4}{*}{\multicolumn{2}{|c|}{ Peak GRF Asymmetry }} \\
\hline & 0.965 & $(0.001)$ & & \\
\hline & 0.958 & $(0.001)$ & & \\
\hline & \multicolumn{2}{|c|}{ Avg GRF Asymmetry } & & \\
\hline & alpha & $\mathrm{p}$ & alpha & $\mathrm{p}$ \\
\hline Overall (\%L-R) & 0.967 & $(<0.001)$ & 0.95 & $(<0.001)$ \\
\hline Overall (\%L-R) R1\&2 & 0.957 & $(0.001)$ & 0.975 & $(0.001)$ \\
\hline Overall (\%L-R) R7\&8 & 0.873 & $(0.007)$ & 0.849 & $(0.012)$ \\
\hline Set1 R1\&2 & 0.908 & $(0.003)$ & 0.936 & $(0.001)$ \\
\hline Set1 R7\&8 & 0.798 & $(0.026)$ & 0.723 & $(0.056)$ \\
\hline
\end{tabular}

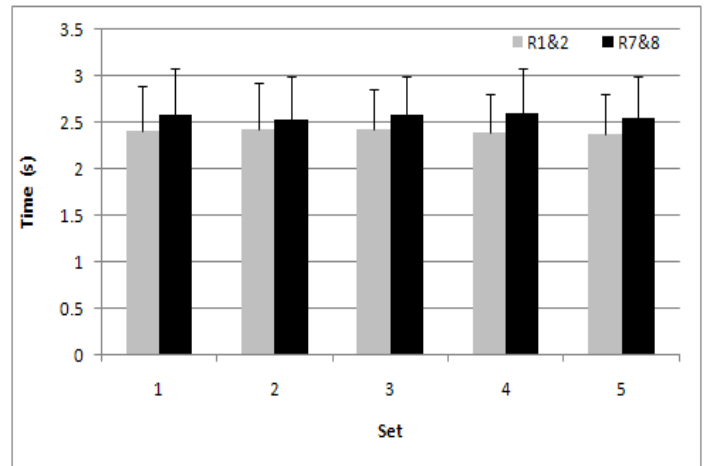

Figure 2: Whole group ( $\mathrm{n}=17)$ average time to complete $\mathrm{R} 1 \& 2$ and $\mathrm{R} 7 \& 8$ of each set with significant main effects occurring for repetition, but not set.

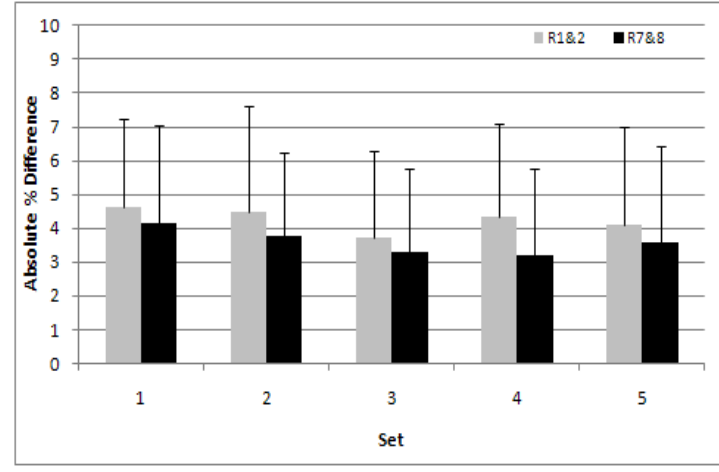

(a)

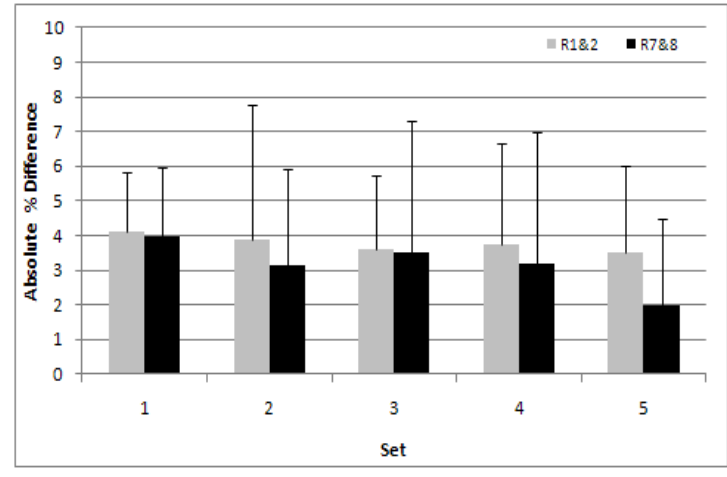

(b)

Figure 3: Whole group absolute average (a) $(n=12)$ and peak $(b)(n=12) \%$ L-R difference with initially symmetric subjects removed. A significant main effect for repetition existed within the average absolute $\%$ L-R asymmetry. 


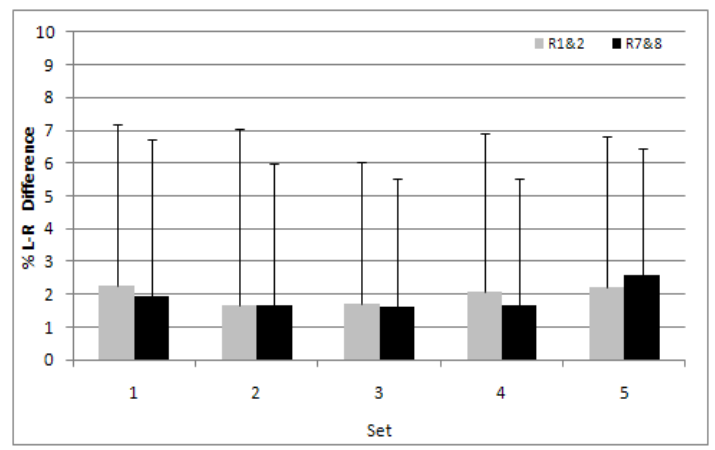

(a)

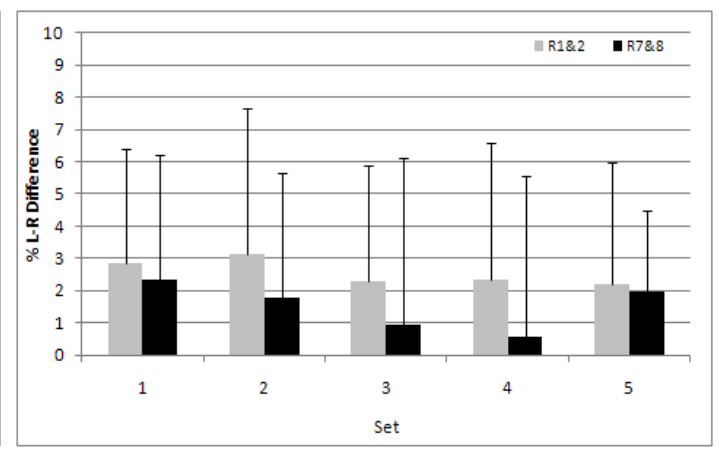

(b)

Figure 4: Whole group average (a) $(n=12)$ and peak (b) $(n=12) \% L-R$ difference with initially symmetric subjects removed. A significant main effect for repetition existed within the peak instantaneous \%L-R asymmetry.

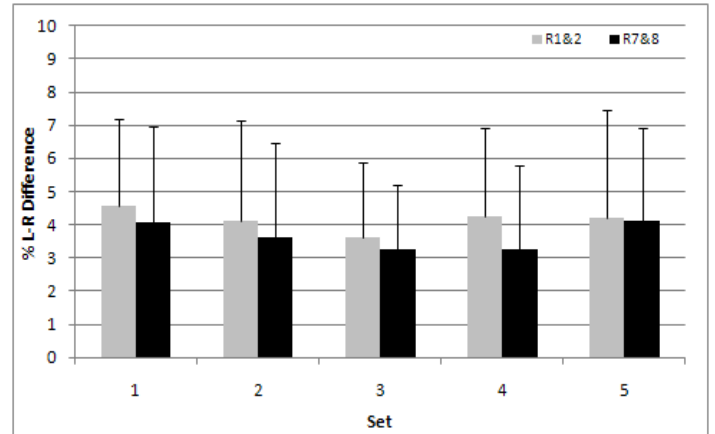

(a)

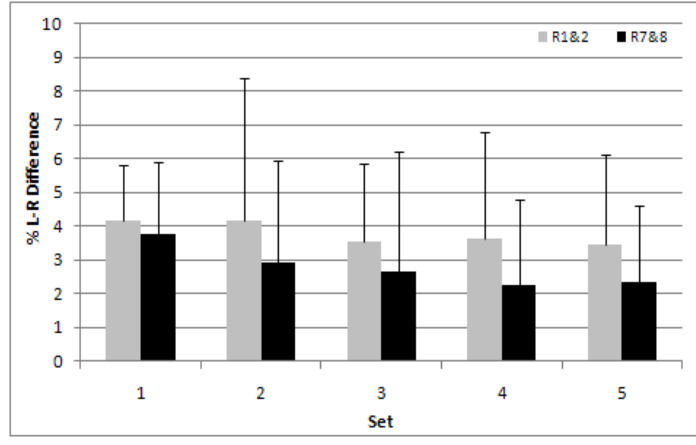

(b)

Figure 5: Whole group average (a) $(n=9)$ and peak (b) $(n=10) \% L-R$ difference, with initially symmetric subjects removed, of those individuals who began with more weight on their left side. While not significant, there was movement towards symmetry in the peak \%L-R difference $(\mathrm{p}=0.08)$.

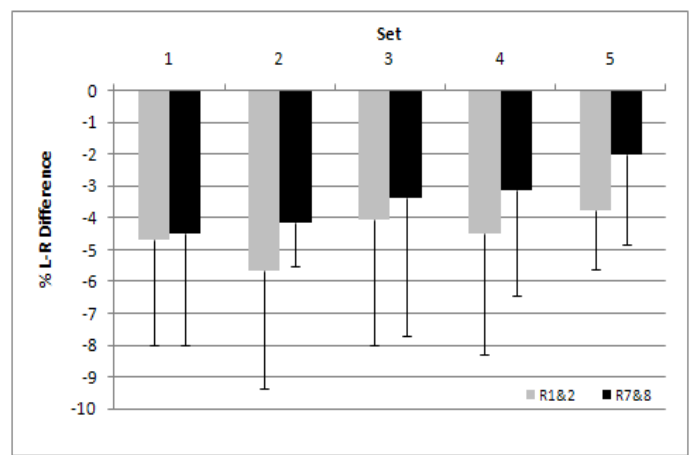

(a)

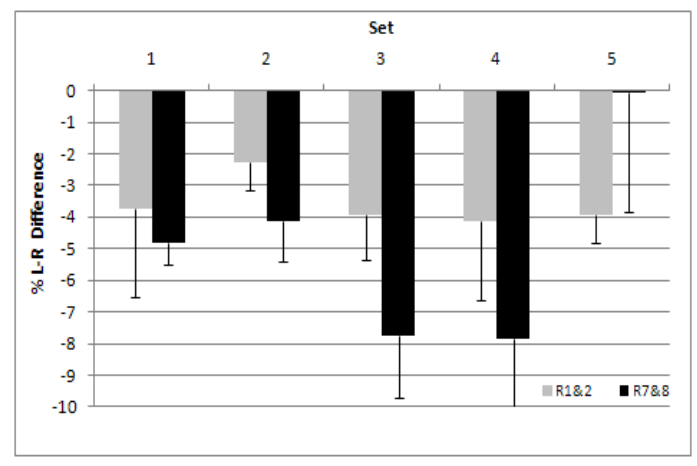

(b)

Figure 6: Whole group average (a) $(n=3)$ and peak (b) $(n=2) \% L-R$ difference, with initially symmetric subjects removed, of those individuals who began with more weight on their right side. A significant main effect for repetition existed within the average \%L-R asymmetry. Note the large SD of R7\&8 of the peak instantaneous graph was cropped (Set $4 \mathrm{R} 7 \& 8 \pm 6.8$ ). 


\section{CHAPTER V}

\section{DISCUSSION}

The goal of this investigation was to explore changes in lower extremity functional asymmetries with a fatiguing free-weight barbell back squat protocol. Functional asymmetries were assessed by individual foot GRFv in a group of healthy, recreationally trained men and women. Asymmetries were examined on the absolute level, not differentiating between left or right side, as well as with consideration for left and right sides. Correlations of anthropometric measures with GRFv asymmetries were also made to understand how geometric bilateral asymmetries may affect functional asymmetries during the fatiguing squat exercise. Assessments were made based on the average and peak instantaneous GRFv of repetitions one and two (R1\&2) and seven and eight (R7\&8) for all five sets. Calculation of both the average and peak instantaneous GRFv asymmetry provides valuable information; for instance, average asymmetry yields a representation of the forces throughout the duration of each repetition, while the peak instantaneous asymmetry reveals the maximum reached during each repetition, which is 
variable, but important to assess changes within and across sets. Fatigue was examined by differences in time to complete each repetition of each set. Finally, day-to-day repeatability was examined.

As expected, even in this healthy population, functional asymmetries were typically present, which is similar to Maines \& Reiser (2006) who found asymmetries in the GRFv of subjects during a submaximal lifting from the floor task. A static lifting task by Rocheford et al. (2006) also revealed subjects favoring one side of the body upon exploration of GRFv when comparing those with previous unilateral injuries to those without. The sit-to-stand task has also evidenced bilateral asymmetries in healthy young and old subjects (Lundin, Grabiner, \& Jahnigen, 1995).

Specific to the squat exercise, Flanagan \& Salem (2007) discovered significant side-to-side differences in bilateral joint moments of 18 recreationally trained men and women during the barbell squat under four loading conditions. Functional asymmetries were also present in the work done by Newton et al. (2006), in which parallel back squats were found to produce significant differences in peak force and average force between dominant and non-dominant legs of 14 collegiate women softball players. Leg dominancy was determined by the strong leg versus the weak leg and not based on the subjects' reported preferred kicking leg. It is reasonable to expect that one leg is stronger than the other, yet this study fails to mention the number of subjects whose strong leg was their preferred kicking leg and vice-versa, however the fact remains that differences were observed. The lack of difference between the right and left legs for the whole group may have been due to the fact that strength differences are nullified when averaged across the group, since some individuals are right-leg dominant and others left-leg dominant, as 
mentioned in their discussion. Similarly, our initial analyses of the whole group ( $\mathrm{n}=17)$ did not reveal significant differences, which may have been due to highly symmetric subjects cancelling out highly asymmetric subjects. Thus, further GRFv asymmetry analyses were conducted with highly symmetric individuals excluded $( \pm 1.7 \%)$.

The only contradictory findings to the general consensus that functional asymmetries exist in healthy populations were the results by Song, Flanagan, Admon, Wang, \& Salem (2003) who discovered symmetric lower extremity joint kinetics in older adults while performing a body weight squat. Clearly external resistance may have been a reason for a lack of observed differences, or the fact that the subjects only performed three trials and only the peak GRFv was analyzed. Typically, as found in our study, people place more weight on the non-kicking leg, as it is known as the stance leg (Gabbard \& Hart, 1996). Thus, it is reasonable to accept that individuals who identify their right leg as their preferred kicking leg would place more weight on their left leg (stance leg) and thus display greater GRFv on the left side when performing tasks.

In a study of unilateral squats, McCurdy \& Langford (2005) also failed to see significant leg strength differences between the dominant and non-dominant legs of healthy men and women. Therefore, isolating legs with unilateral exercises may reduce/remove the dominancy effect. No significant differences between the left and right limbs in amount of torque produced or rate of torque production was seen by Koh, Grabiner, \& Clough (1993) during isometric knee joint extension tasks; however differences were seen in bilateral versus unilateral production with bilateral production being significantly less. 
Within our investigation, asymmetries were relatively consistent for each subject during the workout and were highly repeatable for those that returned for the third visit. This is important to note as this protocol revealed that asymmetries, for the most part, are not random and maintain a consistent expression within and between visits. Maines \& Reiser (2006) also found consistent bilateral differences in GRFv between individual phases, as well as over the course of the entire lift, in a lifting from the floor task. However, they did not test day-to-day variation. The random effects within and across sets of the present study were more typical of highly symmetric individuals, which could be related to balance issues required in the squat exercise, or that fact that highly symmetric individuals were "dancing" around their center of balance. This was evidenced by the fact that switching sides was an anomaly, rather than typical of subjects, indicating that if one starts with more weight on one foot, they were likely to keep weight on that same foot.

Subjects were discouraged from having a "staggered" stance, due to the impact it could play on weight distribution during the squat exercise, and were told to keep their feet planted once beginning a set. However, several subjects were visually observed adjusting their feet throughout the set after initiating R1\&2, which may have added to switching of sides as observed in the data. Staggering foot placement significantly could also affect the magnitude of asymmetry level expressed. With the minimal to nonexistent stagger displayed in our study, levels of asymmetry were low, less than $10 \%$ L-R

The day-to-day repeatability aspect of the current study is similar to that of Hickey et al. (2009) who assessed the repeatability of side-to-side differences in six athletes via the use of a vertical hop power test and the modified agility T-test, both of 
which proved to be reliable assessments between days. Significant differences between the left and right sides on each testing day were not noted, however, they appeared to be rather close based on reported means and standard deviations. Clearly the repeatability aspect of the current study allowed for accurate measurement of individual asymmetries.

The use of a $\pm 1.7 \%$ asymmetry cutoff for the present investigation was chosen arbitrarily to exclude those individuals who were highly symmetric to begin and not expected to change. It has no known relationship to performance or injury risk, although levels ranging from $15-20 \%$ have been established and reported as clinically relevant bilateral strength asymmetries and risk for injury (Impellizzeri et al., 2007; Knapik et al., 1991; Yoshioka et al., 2010). Although methods vary as to the calculation of asymmetry, they usually involve subtracting the weaker leg from the stronger, then dividing by the stronger leg and multiplying by 100 (Impellizzeri et al., 2007), however either the right or left leg can be inserted into the equation as well. Relationships to performance were further suggested by Yoshioka et al., (2010) in that their computer simulation study found no decrease in jump height with $10 \%$ strength asymmetry, but they expected there would be with $20 \%$. The use of a cutoff to exclude subjects with minimal differences between right and left sides has been performed elsewhere in the literature in order to determine if discrepancies between limbs exist (Simon and Ferris, 2008).

In assessing the effects of fatigue during the squat protocol, there was a tendency for subjects to become more symmetric with fatigue, both in the average GRFv and peak instantaneous GRFv, which was contrary to the hypothesis. These changes with fatigue were extremely acute and only existed within a set, not across sets. The observed changes in average GRFv were heavily driven by those that started on their preferred 
kicking leg (right leg) at the beginning of the first set, though not entirely, as this was a small subset of the population. Conversely, it appears those that started on their nonkicking leg (left leg) drove the changes in peak instantaneous GRFv. Why there was a difference between those responsible for the change in average asymmetry versus peak instantaneous asymmetry is not clear. Previous research has examined the effects of fatigue on various jump tasks, such as such as landing from a jump (Pappas et al., 2007; Smith, Sizer, \& James, 2009), performing a drop jump (Moran et al., 2009), performing a maximal vertical jump (Rodacki, Fowler, \& Bennett, 2002), and single-leg hop testing (Augustsson et al., 2006), but is limited on the effects of fatigue during the squat exercise, as well as how fatigue influences asymmetries. The majority of the literature examines fatigue in the context of reduced forces, moments, joint angles, jump height, or jump distance, and does not compare side-to-side differences to assess asymmetries. Therefore, it is difficult to relate the findings of this study to the work of others. It is warranted to discuss the findings of Carpes et al., (2007), as they revealed that pedaling asymmetries decreased as crank torque and cycling exercise intensity $\left(\% \mathrm{VO}_{2} \max \right)$ increased. Intensity relative to $\% \mathrm{VO}_{2} \max$ may then be a valid surrogate for fatigue, which is in line with the present study, as well as the work by Flanagan \& Salem (2007) with the squat exercise, that asymmetries stay the same or decrease with fatigue.

It should however be noted that Oda \& Moritani (1995) demonstrated a difference in force decline, which was greater for the left arm, when comparing right and left arms of 25 right-handed oarsmen who performed a sustained maximal voluntary elbow flexion exercise. Differences in external power output between the arms, which was greater for the left, of swimmers during swim bench exercises have also been observed (Potts et al., 
2002). Further, the magnitude of difference in bilateral arm power increased as subjects reached exhaustion. These groups (oarsmen and swimmers) however, were expected to be asymmetric based on their training.

Despite the fact that these two findings were based on the upper extremities, it is imaginable that such differences may exist in the lower extremities as well. Perhaps becoming more symmetric with fatigue in the present study was a result of use of a bilateral exercise, which necessitated joint effort from the lower extremities to maintain balance. Simon \& Ferris (2008), in a study assessing 10 subjects who performed lower limb isometric extensions on an exercise machine, discovered that activating the legs at the same time while performing bilateral movements lead to asymmetric limb forces, concluding that asymmetries are neurologically based, rather than mechanically based. The greater differences seen at the start of a set in the current study may have been due to subjects figuring out how to activate the lower limbs at the same time, while getting better at it through the course of a set, where greater symmetry was seen, thus a sort of "practice effect" occurred. This may also be why it did not carry over from set to set.

Regarding anthropometric measurements, the study population was highly symmetric. Accurate measurements were taken, as the abilities of the researchers to repeat the anthropometric measurements were quite high from visit-to-visit, although not at the level to consistently reproduce side-to-side differences. This may be due to the fact that such small differences between the right and left sides existed and any deviation from visit-to-visit produced large deviations in the data. Of the measures that were more repeatable, there was greater correlation with functional GRFv asymmetries, suggesting that there is a link between the two. This is a reasonable finding in that difference in limb 
usage has been reported (White et al., 2004), such that the shorter limb accepts more peak GRFv and higher rates of loading than the longer limb in individuals with a true or simulated leg length inequality. Krwczyk et al. (1998) discovered lower right-left differences in anthropometric measurements of athletes engaging in sports requiring symmetric movements compared to those playing sports necessitating asymmetric movements identified through the measurements of 134 elite male athletes from nine different sports. While functional asymmetries were not directly examined in the above mentioned study, it is reasonable to think that larger right-left differences based on anthropometric measurements would lead to larger right-left functional asymmetries, especially when considering individuals who routinely perform movements either symmetrically or asymmetrically. While anthropometric measures were not performed in the study by Simon \& Ferris (2008), they did however reveal that limb force asymmetry was related to neural factors instead of mechanical capability differences between the limbs. The relationship between neural factors and anthropometric asymmetry is not understood.

As stated earlier, differences were seen within sets, but not across sets. Perhaps, if the rest time between sets was reduced, changes across sets and not just within reps may appear. Clearly the findings indicate that fatigue was induced within a set, as supported by the time to complete repetitions, but did not heavily transfer from set to set. Adequate rest was given between sets as evidenced by the fact that time to complete repetitions did not increase across sets and all subjects completed all eight repetitions of all five sets. Also likely is the fact that $90 \%$ of a subjects' 8 RM was used, to ensure completion of the protocol, and using the actual 8 RM may have yielded significant 
differences in time to complete each set. It is also possible that some subjects may not have reached a true 8 RM on visit number one. A review by Sale (1991) discussed the issue that it is not uncommon for re-test results to differ from initial repetition maximum results and that subject's strength scores may increase when tests are repeated. The fact that subjects fatigued during a set, evidenced by taking more time to complete R7\&8 compared to R1\&2, as well as became more symmetric, relates to a finding by Koh et al. (1993) who found a larger bilateral deficit when subjects were instructed to generate force rapidly rather than gradually. Perhaps the increased repetition time lead to decreased asymmetries in the current study, as subjects generated force at a slightly reduced pace.

Limitations of the present study include a relatively small sample size and a homogenous population, which prohibited the ability to assess left-handed/left-footed subjects. Additionally, kinematics and muscle activation data would have allowed for further understanding of the sources of side-to-side differences. For instance, assessment of net joint torques of the lower extremities from side-to-side would have allowed the identification of different contributions of the lower limbs in performing the squat exercise and how they change with fatigue. Muscle activation data would have also allowed for the assessment of muscle force generation with fatigue and how it may differ from side-to-side. However, as Lawson et al. (2006) and Stephens et al. (2007) reported, finding consistent sources of asymmetry may be quite challenging. Further, assessment of forces in more than one plane, such as medial/lateral (GRFm/l) and anterior/posterior (GRFa/p), would have allowed for additional understanding of asymmetries during the squat exercise. Yet Maines \& Reiser (2006) reported inconsistent GRFa/p in a crate- 
lifting task, stating that it may be due to the purpose of the force (balance in direction of the base of support), as Giakas, Baltzopoulous, Dangerfield, Dorgan, \& Dalmira (1996) found GRFa/p to be highly variable between individuals and tasks.

Additional limitations include the fact that subjects were instructed to refrain from intense lower extremity exercise 48 hours prior to each visit and were taken at their word for self-reported behavior. Further, appropriate subjects were deemed acceptable based on their self-reported participation in a lifting program and weekly jump utilization.

In terms of practical applications, low levels of functional asymmetries do not appear to increase during highly controlled bilateral exercises, such as the free-weight squat. If anything, a person tends to become slightly more symmetric during the course of the exercise. Therefore, both legs should be stressed similarly by the exercise. Thus, bilateral exercises should continue to be incorporated into strength training programs. While unilateral training may not be warranted based on these results for strength development, unilateral training may still be desirable for development of single-leg coordination and the development of muscles not specifically targeted by bilateral squats, such as those required for medial/lateral control of the hip, knee, and ankle.

It is unclear, based on the findings of the current study, how this translates beyond free-weight squats in healthy, recreationally trained subjects. For example, those with significant levels of asymmetry may respond differently, as may those who have no previous weight training experience. Since the use of a free-weight squat requires significant balance, exercises where a balance component is not present may present different results (i.e., Smith machine squats or leg presses). Furthermore, the current 
study was performed in a controlled environment, so it is uncertain how more dynamic movements, such as sport play, may allow a person to favor one side more than the other, effecting fatigue and asymmetry levels.

In conclusion, low levels of relatively consistent functional asymmetries exist in healthy, recreationally trained men and women while performing a free-weight barbell back squat. That fact that subjects became more symmetric with fatigue should not deter strength and conditioning coaches from incorporating this exercise in training programs. Rather, this supports their value for bilateral development in healthy subjects with no known reason for being asymmetric. Finally, future research is needed to understand the role fatigue plays during the course of other exercises and various populations on asymmetries, as well as how these translate to sport performance and risk of injury. 


\section{REFERENCES}

Arendt, E., \& Griffin, L. (2000). Musculoskeletal injuries. In B.L. Drinkwater (Ed.), Women in Sport (pp. 208-240). Malden, MA: Blackwell Sciences, Ltd.

Augustsson, J., Thomee, R., Linden, C., Folkesson, M., Tranberg, R., \& Karlsson, J. (2006). Single-leg hop testing following fatiguing exercise: reliability and biomechanical analysis. Scandinavian Journal of Medicine and Science in Sports, 16: 111-120.

Beelen, A., \& Sargeant, A.J. (1993). Effect of prior exercise at different pedaling frequencies on maximal power in humans. European Journal of Applied Physiology, 66: 102-107.

Blaszczyk, J.W., Prince, F., Raiche, M., \& Hebert, R. (2000). Effect of ageing and vision on limb load asymmetry during quiet stance. Journal of Biomechanices, 33: $1243-1248$.

Blustein, S.M., \& D’Amico, J.C. (1985). Limb length discrepancy: identification, clinical significance, and management. Journal of the American Podiatric Medical Association, 75(4): 200-206.

Carpes, F.P., Rossato, M., Faria, I.E., \& Bolli Mota, C. (2007). Bilateral pedaling asymmetry during a simulated $40-\mathrm{km}$ cycling time-trial. The Journal of Sports Medicine and Physical Fitness, 47(1): 51-57.

Challis, J.H. (1998). An investigation of the influence of bi-lateral deficit on human jumping. Human Movement Science, 17: 307-325.

Chandler, T.J., \& Stone, M.H. (1991). The squat exercise in athletic conditioning: a review of the literature. National Strength and Conditioning Association Journal, 13(5): 51-60.

Daly, D.J., \& Cavanagh, P.R. (1976). Asymmetry in bicycle ergometer pedalling. Medicine and Science in Sports, 8(3): 204-208.

Delacerda, F.G., \& McCrory, M.L. (1981). A case report: effect of a leg length differential on oxygen consuption. The Journal of Orthopaedic and Sports Physical Therapy, 3(1): 17-20.

Dugan, S.A., \& Frontera, W.R. (2000). Muscle fatigue and muscle injury. Scientific Principles of Sports Rehabilitation, 11(2): 385-403.

Escamilla, R.F. (2001). Knee biomechanics of the dynamic squat exercise. Medicine and Science in Sports and Exercise, 33(1): 127-141.

Evans, R.C. (1994). Illustrated essentials in orthopedic physical assessment. St. Louis, MO: Mosby-Year Book, Inc. 
Ewing, J.L., \& Stull, G.A. (1984). Rate of force development in the handgripping muscles by females as a function of fatigue level. Research Quarterly for Exercise and Sport, 55(1): 17-23.

Ferber, R., Osternig, L.R., Woollacott, M.H., Wasielewski, N.J., \& Lee, J.H. (2004). Bilateral accommodations to anterior cruciate ligament deficiency and surgery. Clinical Biomechanics, 19: 136-144.

Flanagan, S.P., \& Salem, G.J. (2007). Bilateral differences in the net joint torques during the squat exercise. Journal of Strength and Conditioning Research, 21(4): 12201226.

Giakas, G., Baltzopoulos, V., Dangerfield, P.H., Dorgan, J.C., Dalmira, S. (1996). Comparison of gait patterns between health and scoliotic patients using time and frequency domain analysis of ground reaction forces. Spine, 21: 2235-2242.

Gabbard, C., \& Hart, S. (1996). A question of foot dominance. The Journal of General Psychology, 123(4): 289-296.

Gupta, S., Nicholson, L., Shirley, D., \& Adams, R. (2006). Asymmetry of muscle activation in a symmetrical, bilateral lower limb. Journal of Science and Medicine in Sport, 9: 34-35.

Hakkinen, K. (1993). Neuromuscular fatigue and recovery in male and female athletes during heavy resistance exercise. International Journal of Sports Medicine, 14(2): 53-59.

Hattin, H.C., Pierrynowski, M.R., \& Ball, K.A. (1989). Effect of load, cadence, and fatigue on tibiofemoral joint force during a half squat. Medicine and Science in Sports and Exercise, 21(5): 613-618.

Hennessy, L.C., \& Watson, A. W. S. (1994). The interference effects of training for strength and endurance simultaneously. Journal of Strength and Conditioning Research, 8(1): 12-19.

Hewett, T.E., Lindenfeld, T.N., Riccobene, J.V., \& Noyes, F.R. (1999). The effect of neuromuscular training on the incidence of knee injury in female athletes. The American Journal of Sports Medicine, 27(6): 699-706.

Hewett, T.E., Stroupe, A.L., Nance, T.A., \& Noyes, F.R. (1996). Plyometric training in female athletes: decreased impact forces and increased hamstring torques. The American Journal of Sports Medicine, 24(6): 765-773.

Hickey, K.C., Quatman, C.E., Myer, G.D., Ford, K.R., Brosky, J.A., \& Hewett, T.E. (2009). Methodological report: dynamic field tests used in an NFL combine setting to identify lower-extremity functional asymmetries. The Journal of Strength and Conditioning Research, 23(9): 2500-2506.

Hinson, R., \& Brown, S.H. (1998). Supine leg length differential estimation: an inter-and intra-examiner reliability study. Chiropractic Research Journal, 5(1): 17-22. 
Hoeger, W. W. K., Hopkins, D. R., Barette, S.L., \& Hale, D.F. (1990). Relationship between repetitions and selected percentages of one repetition maximum: a comparison between untrained and trained males and females. Journal of Applied Sport Science Research, 4(2): 47-54.

Impellizzeri, F.M., Rampinini, E., Maffiuletti, N., \& Marcora, S.M. (2007). A vertical jump force test for assessing bilateral strength asymmetry in athletes. Medicine and Science in Sports and Exercise, 39(11): 2044-2050.

Johnston, R.B., Howard, M.E., Cawley, P.W., \& Losse, G.M. (1998). Effect of lower extremity muscular fatigue on motor control performance. Medicine and Science in Sports and Exercise, 30(12): 1703-1707.

Kearney, J.T, \& Stull, G.A. (1981). Effect of fatigue level on rate of force development by the grip-flexor muscles. Medicine and Science in Sports and Exercise, 13(5): 339-342.

Knapik, J.J., Bauman, C.L., Jones, B.H., Harris, J. McA., \& Vaughan, L. (1991). Preseason strength and flexibility imbalances associated with athletic injuries in female collegiate athletes. The American Journal of Sports Medicine, 19(1): 7681.

Knutson, G.A. (2005). Anatomic and functional leg-length inequality: a review and recommendation for clinical decision-making. Part I, anatomic leg-length inequality: prevalence, magnitude, effects and clinical significance. Chiropractic and Osteopathy, 13(11): 1-10.

Koh, T.J., Grabiner, M.D., and Clough, C.A. (1993). Bilateral deficit is larger for step than for ramp isometric contractions. Journal of Applied Physiology, 74 (3): $1200-1205$.

Krawczyk, B., Sklad, M., Majle, B., \& Jackiewicz, A. (1998). Lateral asymmetry in upper and lower limb measurements in selected groups of male athletes. Biology of Sport, 15(1): 33-38.

Kujala, U.M., Friberg, O., Aalto, T., Kvist, M., \& Osterman, K. (1987). Lower limb asymmetry and patellofemoral joint incongruence in the etiology of knee exertion injuries in athletes. International Journal of Sports Medicine, 8: 214-220.

Lawson, B.R., Stephens, T.M., DeVoe, D.E., \& Reiser, R.F. (2006). Lower-extremity bilateral differences during step-close and no-step countermovement jumps with concern for gender. Journal of Strength and Conditioning Research, 20(3): 608619.

Leroy, D., Polin, D., Tourny-Chollet, C., \& Weber, J. (2000). Spatial and temporal gait variable differences between basketball, swimming and soccer players. International Journal of Sports Medicine, 21: 158-162. 
Maines, J.M., \& Reiser, R.F. (2006). Ground reaction force bilateral asymmetries during submaximal sagittal plane lifting from the floor. International Journal of Industrial Ergonomics, 36: 109-117.

Manning, J.T., \& Pickup, L.J. (1998). Symmetry and performance in middle distance runners. Internation Journal of Sports Medicine, 19: 205-209

McCurdy, K., \& Langford, G. (2005). Comparison of unilateral squat strength between the dominant and non-dominant leg in men and women. Journal of Sports Science and Medicine, 4: 153-159.

McLean, B.D., \& Tumilty, D. McA. (1993). Left-right asymmetry in two types of soccer kick. British Journal of Sports Medicine, 27(4): 260-262.

McLean, S.G., \& Samorezov, J.E. (2009). Fatigue-induced ACL injury risk stems from a degradation in central control. Medicine and Science in Sports and Exercise, 41(8): 1661-1672.

Neitzel, J.A., Kernozek, T.W., \& Davies, G.J. (2002). Loading response following anterior cruciate ligament reconstruction during the parallel squat exercise. Clinical Biomechanics, 17: 551-554.

Newton, R.U., Gerber, A., Nimphius, S., Shim, J.K., Doan, B.K., Robertson, M., Pearson, D.R., Craig, B.W., Hakkinen, K., \& Kraemer, W.J. (2006). Determination of functional strength imbalance of the lower extremitites. Journal of Strength and Conditioning Research, 20(4): 971-977.

Oda, S., \& Moritani, T. (1995). Cross-correlation of bilateral differences in fatigue during sustained maximal voluntary contraction. European Journal of Applied Physiology, 70: 305-310.

Pappas, E., Sheikhzadeh, A., Hagins, M., \& Nordin, M. (2007). The effect of gender and fatigue on the biomechanics of bilateral landings from a jump: peak values. Journal of Sports Science and Medicine, 6: 77-84.

Potts, A.D., Charlton, J.E., \& Smith, H.M. (2002). Bilateral arm power imbalance in swim bench exercise to exhaustion. Journal of Sports Sciences, 20: 975-979.

Rocheford, E.C., DeVoe, D.E., \& Reiser, R.F. (2006). Effect of previous unilateral injuries on ground reaction force bilateral asymmetries during static lifting and standing. Journal of Human Movement Studies, 51: 403-424.

Rodacki, A.L.F., Fowler, N.E., \& Bennett, S.J. (2002). Vertical jump coordination: fatigue effects. Medicine and Science in Sports and Exercise, 34(1): 105-116.

Sadeghi, H., Allard, P., Prince, F., \& Labelle, H. (2000). Symmetry and limb dominance in able-bodied gait: a review. Gait and Posture, 12: 34-45. 
Sale, D.G. (1991). Testing strength and power. In J.D. MacDougall, H.A. Wenger, \& H.J. Green (Eds.), Physiological testing of the high-performance athlete (pp. 21106). Champaign: Human Kinetics.

Salem, G.J., Salinas, R., \& Harding, F.V. (2003). Bilateral kinematic and kinetic analysis of the squat exercise after anterior cruciate ligament reconstruction. Archives of Physical Medicine and Rehabilitation, 84: 1211-1216.

Salvador, E.P., Dias, R.M.R., Gurjao, A.L.D., Avelar, A., Pinto, L.G., \& Cyrino, E.S. (2009). Effect of eight weeks of strength training on fatigue resistance in men and women. Isokinetics and Exercise Science, 17: 101-106.

Sewall, L.P., \& Lander, J.E. (1991). The effects of rest on maximal efforts in the squat and bench press. Journal of Applied Sport Science Research, 5(2): 96-99.

Schiltz, M., Lehance, C., Maquet, D., Bury, T., Crielaard, J.M., \& Croisier, J.L. (2009). Explosive strength imbalances in professional basketball players. Journal of Athletic Training, 44(1): 39-47.

Shaw, C.N., \& Stock, J.T. (2009). Habitual throwing and swimming correspond with upper limb diaphyseal strength and shape in modern human athletes. American Journal of Physical Anthropology, 140: 160-172.

Simon, A.M., \& Ferris, D.P. (2008). Lower limb force production and bilateral force asymmetries are based on sense of effort. Experimental Brain Research, 187: 129-138.

Singh, I. (1970). Functional asymmetry in the lower limbs. Acta anatomica, 77: 131-138.

Smak, W., Neptune, R.R., \& Hull, M.L. (1999). The influence of pedaling rate on bilateral asymmetry in cycling. Journal of Biomechanics, 32(9): 899-906.

Smith, M.P., Sizer, P.S., \& James, C.R. (2009). Effects of fatigue on frontal plane knee motion, muscle activity, and ground reaction forces in men and women during landing. Journal of Sports Science and Medicine, 8: 419-427.

Stephens, T.M., Lawson, B.R., DeVoe, D.E., \& Reiser, R.F. (2007). Gender and bilateral differences in single-leg countermovement jump performance with comparison to a double-leg jump. Journal of Applied Biomechanics, 23: 190-202.

Tomkinson, G.T., Popovic, N., \& Martin, M. (2003). Bilateral symmetry and the competitive standard attained in elite and sub-elite sport. Journal of Sport Sciences, 21: 201-211.

Trivers, R., Manning, J.T., Thornhill, R., Singh, D., \& McGuire, M. (1999). Jamaican symmetry project: long-term study of fluctuating asymmetry in rural Jamaican children. Human Biology, 71: 417-430.

Vila-Cha, C., Carvalho, J.M., Machado, L., Conceicao, F., \& Vilas Boas, J.P. (2005). 
Associations between biomechanics pattern changes and muscle fatigue during prolonged parallel half squat series (abstract). British Journal of Sports Medicine, 39(6): 404.

White, S.C., Gilchrist, L.A., \& Wilk, B.E. (2004). Asymmetric limb loading with true or simulated leg-length differences. Clinical Orthopaedics and Related Research, 421: 287-292.

Yoshioka, S., Nagano, A., Hay, D., \& Fukashiro, S. (2010). The effect of bilateral asymmetry of muscle strength on jumping height of the countemovment jump: A computer simulation study. Journal of Sports Sciences, 28(2): 209-218. 
APPENDICES 


\section{APPENDIX A}

Human Subjects Approval 
Research Integrity \& Compllance Review Office

Omlice of the Vice President for Research

321 General Services Bullding - Campus Dellvery 2011

Fort Collins, co

TEL:H(970) 491-1553

FAX:\#(970) 491-2293

\section{NOTICE OF APPROVAL FOR HUMAN RESEARCH}

DATE:

TO:

FROM:

PROTOCOL TITLE:

FUNDING SOURCE:

PROTOCOL NUMBER:

APPROVAL PERIOD:
September 29, 2009

Rairsc, Ravol, Ph. D., Health \& Exarcise Science

Istael, Richard, EdD, Heslth \& Exarcine Science, Patrick, Ryan, Hevlth \& Exercise Scieece, Hodgos, Stephamie, Heslth \& Exercise Science

Burker, Janell, CSU IRB 1

Effact of fatigue on jump and squzt asymmetrios

NONE

09-1358H

Approval Date: September 29, $2009 \quad$ Explration Date: September 24, 2010

The CSU Institutional Review Board (IRB) for the protection of human subjects has revlewed the protocol enittlect: Ellect of fatigue on jump and squat asymmetries. The project has been approved for the procedures and subjects described in the protocol. This protocol must be revlewed for renewal on a yearly basis for as long as the research remains active. Should the protocol not be renewed before explration, all activities must cease until the protocol has been re-reviewed.

If approval did not accompany a proposal when It was submitted to a sponsor, It is the PI's responsibilty to provide the sponsor with the approval notice.

This approval Is Issued under Colorado State Unlversity's Federal Wide Assurance 00000647 with the Omice for Human Research Protections (OHRP). If you have any questions regarding your obligations under CSU's Assurance, please do not hesitate to contact us.

Please direct any questions about the IRB's actions on this project to:

Janell Barker, Senlor IRB Coordlnator - (970) 491-1655 JanellBarker@Research.Colostate.edu

Evelyn Swlss, IRB Coordlnator - (970) 491-1381 Evelyn.Swss:BResearch.Colostate.edu

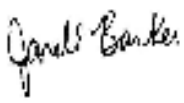

Barker, Janell

Includes: Approval is for a maximum of 30 participants between the ages of 18-30 using the approved consent form with the CONDITION that the two methods sections are submitted once available and uploaded as an amendment.

Approval Perlod:

Review Type:

IRB Number:
September 29, 2009 through September 24, 2010

EXPEDITED

00000202 
APPENDIX B

Consent Form 


\title{
Consent to Participate in a Research Study \\ Colorado State University
}

TITLE OF STUDY: Effect of fatigue on jump and squat asymmetries

\author{
PRINCIPAL INVESTIGATOR: Raoul F. Reiser, II \\ Contact Information: 970-491-7980,RFReiser@CAHS.Colostate.edu
}

\begin{abstract}
WHY AM I BEING INVITED TO TAKE PART IN THIS RESEARCH? You are being asked to volunteer for this research because you are a relatively fit healthy adult between the ages of 18-30 years with no current injuries (pain and soreness free for the last month). You must also be free of any current or chronic low-back pain, leg pain, or orthopedic problems. You must be able to perform a series of jumping tasks, as well as multiple sets of your 8 repetition maximum (8RM) free-weight squat.
\end{abstract}

WHO IS DOING THE STUDY? This research is being performed by Raoul F. Reiser II, Ph.D. of the Health and Exercise Science Department. Trained graduate students, undergraduate students, research associates, or research assistants are assisting with the research.

WHAT IS THE PURPOSE OF THIS STUDY? It is well documented that asymmetries exist in the lower extremity (i.e., one leg is favored over the other). However, it is not clear if those asymmetries change with fatigue and how fatigue might affect performance. The goal of this investigation is to determine if and how asymmetries change with fatigue.

WHERE IS THE STUDY GOING TO TAKE PLACE AND HOW LONG WILL IT LAST? This research project will take place in the Clinical Biomechanics Laboratory located on the $2^{\text {nd }}$ floor of Moby Arena (B wing) on the CSU main campus. Your involvement will last roughly 1 hour each on 2 separate occasions. If you return for repeat visits, to help us determine the reliability of our measures, your involvement will double.

WHAT WILL I BE ASKED TO DO? If you agree to participate we will check your jump and squat technique and verify/determine your $8 R M$ on the first visit. On the second visit you will be required to perform a series of jumps pre- and post-fatigue. There will be maximum effort vertical jumps using both feet as well as short single-leg forward hops. Five sets of 8RM squats will be performed in between the pre-and post-fatigue jumping conditions. While performing all tasks, the forces under your feet will be measured. There are no needles or other devices that will break the skin. We will lead you through a warm-up procedure, have you practice the task at submaximal levels of effort, spot you during the jumps and squats, and give you rest between efforts.

ARE THERE REASONS WHY I SHOULD NOT TAKE PART IN THIS STUDY? You should not volunteer for this study if you do not meet the criteria outlined above. Additionally, if you have any preexisting conditions that would lead you to believe that you are highly 
asymmetric, you should not participate. If you are a woman, you should not participate if you are pregnant. Regardless of gender, you should also not participate if you have any reason to believe you might be injured by these activities. All physical exertions are controlled by you. The amount you will squat will be based on determination of your 8RM, performed during your first visit.

WHAT ARE THE POSSIBLE RISKS AND DISCOMFORTS? As with all physical activity, there is a risk for injury. The most likely risks associated with this study are muscle strains and pulls as well as muscle fatigue. That fact that you are relatively fit, are familiar with the squat and jump, and that we will spot you and give you plenty of opportunity to stretch and warm-up minimizes these risks.

You are also given breaks between each squat trials to minimize risk for injury. If at any time you feel uncomfortable, pain, or are excessively tired, you should discontinue effort and tell the investigator. It is not possible to identify all potential risks in research procedures, but the researcher has taken reasonable safeguards to minimize any known and potential, but unknown, risks.

WILL I BENEFIT FROM TAKING PART IN THIS STUDY? While this study should provide useful information that may in the future provide useful information on lower limb asymmetries and fatigue, there are no current benefits to participation in this study.

DO I HAVE TO TAKE PART IN THE STUDY? Your participation in this research is voluntary. If you decide to participate in the study, you may withdraw your consent and stop participating at any time without penalty or loss of benefits to which you are otherwise entitled.

WHAT WILL IT COST ME TO PARTICIPATE? There are no costs to participate in this study.

WHO WILL SEE THE INFORMATION THAT I GIVE? We will keep private all research records that identify you, to the extent allowed by law.

Your information will be combined with information from other people taking part in the study. When we write about the study to share it with other researchers, we will write about the combined information we have gathered. You will not be identified in these written materials. We may publish the results of this study; however, we will keep your name and other identifying information private.

We will make every effort to prevent anyone who is not on the research team from knowing that you gave us information, or what that information is. For example, your name will be kept separate from your research records and these two things will be stored in different places under lock and key. You should know, however, that there are some circumstances in which we may have to show your information to other people. For example, the law may require us to show your information to a court. The files containing information about you will be identified with a code, such as "FS01", where FS is short for Fatigue Subject and 01 is a subject number. Upon completion of data collection and verification of results, the list linking your name to the code will be destroyed.

CAN MY TAKING PART IN THE STUDY END EARLY? Your participation in the study may end early if we are unable to measure ground reaction forces during jumping or squatting 
tasks. This is usually due to equipment error, but may also occur for unknown reasons. The study may also end early if you experience any pain or discomfort, or are unable to complete the jump and squat protocols.

WILL I RECEIVE ANY COMPENSATION FOR TAKING PART IN THIS STUDY? There is no monetary compensation for your involvement in the study. However, if you are a student in an HES activity course (HES 1000, HES 100N, or HES 332F) your participation qualifies you for "extra credit". Speak with your course instructor for details.

WHAT HAPPENS IF I AM INJURED BECAUSE OF THE RESEARCH? The Colorado Governmental Immunity Act determines and may limit Colorado State University's legal responsibility if an injury happens because of this study. Claims against the University must be filed within 180 days of the injury.

In light of these laws, you are encouraged to evaluate your own health and disability insurance to determine whether you are covered for any physical injuries or emotional distresses you might sustain by participating in this research, since it may be necessary for you to rely on your individual coverage for any such injuries. Some health care coverages will not cover research-related expenses. If you sustain injuries, which you believe were caused by Colorado State University or its employees, we advise you to consult an attorney.

WHAT IF I HAVE QUESTIONS? Before you decide whether to accept this invitation to take part in the study, please ask any questions that might come to mind now. Later, if you have questions about the study, you can contact the investigator, Raoul F. Reiser II, Ph.D. at 970-491-6958. If you have any questions about your rights as a volunteer in this research, contact Janell Barker, Human Research Administrator at 970-491-1655. We will give you a copy of this consent form to take with you.

Your signature acknowledges that you have read the information stated and willingly sign this consent form. Your signature also acknowledges that you have received, on the date signed, a copy of this document containing 3 pages.

Signature of person agreeing to take part in the study

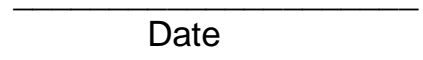

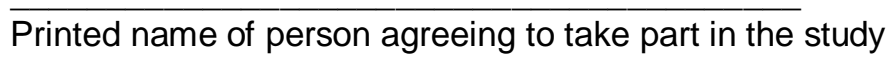

Name of person providing information to participant

\section{Date}

Signature of Research Staff 
APPENDIX C

Health and Activity Questionnaire 


\section{Effect of Fatigue on Jump \& Squat Asymmetries}

(Fall-Spring 2009-10)

Health \& Activity Screening - Coded Cover Sheet

(Separate from the coded screening form, store separately)

Name (Last, First):

Address:

Phone number:

Email:

Code \#:

Acceptable Subject: Yes or No

Notes 


\section{Health \& Activity Questionnaire}

Code \#

Are you between the ages of 18-30 years?

Are you healthy (pain, soreness, and injury free)?

Women only: Are you pregnant?

Do you have now, or ever had, chronic low-back pain or scoliosis?

Do you have any orthopedic or arthritic problems?

Do you have any prior injuries or conditions that would cause you to favor one side of the body while performing otherwise symmetric tasks (broken bones, torn muscles and ligaments, a leg-length differential, ACL reconstruction etc.)?

Do you use any bilateral corrective devices (heel lift, brace, etc.)?

Have you participated in a resistance-training program, which includes a free weight squat for at least the past 8 weeks?

Do you participate in sports that involve jumping on a weekly basis?

Are you comfortable exerting high levels of effort from the low back?

Are you comfortable jumping or landing with moderate fatigue?

Any current or chronic medical conditions (heart disease, diabetes, asthma, allergies, epilepsy, etc.) or medications that would prevent or interfere with maximal physical activity?

Please estimate your Height:___ Weight:

Squat 8 RM: 
Should you be identified as a good match for this project based on your answers, can we schedule a $1 \mathrm{hr}$ block of time for you to come in to the lab?

Should you be scheduled for a visit to the lab to participate in the study, will you refrain from major physical activity using the legs 48 hours prior to lab visits and refrain from excessive caffeine and/or ergogenic aids on the days of the lab visit?

Should you be asked to participate in this study, do you agree to wear clothing that does not restrict motion ( $\mathrm{t}$-shirt, shorts, comfortable shoes, without excessive heel lift, that would be appropriate for squatting and jumping tasks)? 